\title{
Rewriting and narrowing for constructor systems with call-time choice semantics*
}

\author{
FRANCISCO J. LÓPEZ-FRAGUAS, ENRIQUE MARTIN-MARTIN, \\ JUAN RODRÍGUEZ-HORTALÁ and JAIME SÁNCHEZ-HERNÁNDEZ \\ Departamento de Sistemas Informáticos y Computación \\ Universidad Complutense de Madrid, Spain \\ (e-mail: fraguas@sip.ucm.es, emartinm@fdi.ucm.es, \\ juan.rodriguez.hortala@gmail.com, jaime@sip.ucm.es)
}

submitted 30 November 2010; revised 14 November 2011; accepted 4 July 2012

\begin{abstract}
Non-confluent and non-terminating constructor-based term rewrite systems are useful for the purpose of specification and programming. In particular, existing functional logic languages use such kind of rewrite systems to define possibly non-strict non-deterministic functions. The semantics adopted for non-determinism is call-time choice, whose combination with non-strictness is a non trivial issue, addressed years ago from a semantic point of view with the Constructor-based Rewriting Logic (CRWL), a well-known semantic framework commonly accepted as suitable semantic basis of modern functional logic languages. A drawback of CRWL is that it does not come with a proper notion of one-step reduction, which would be very useful to understand and reason about how computations proceed. In this paper we develop thoroughly the theory for the first order version of letrewriting, a simple reduction notion close to that of classical term rewriting, but extended with a let-binding construction to adequately express the combination of call-time choice with non-strict semantics. Let-rewriting can be seen as a particular textual presentation of term graph rewriting. We investigate the properties of let-rewriting, most remarkably their equivalence with respect to a conservative extension of the CRWL-semantics coping with let-bindings, and we show by some case studies that having two interchangeable formal views (reduction/semantics) of the same language is a powerful reasoning tool. After that, we provide a notion of let-narrowing which is adequate for call-time choice as proved by soundness and completeness results of let-narrowing with respect to letrewriting. Moreover, we relate those let-rewriting and let-narrowing relations (and hence CRWL) with ordinary term rewriting and narrowing, providing in particular soundness and completeness of let-rewriting with respect to term rewriting for a class of programs which are deterministic in a semantic sense.
\end{abstract}

\section{Introduction}

Term rewriting systems (TRS, (Baader and Nipkow 1998)) are a well-known and useful formalism from the point of view of specification and programming. The

\footnotetext{
* This work has been partially supported by the Spanish projects FAST-STAMP (TIN2008-06622C03-01/TIN), PROMETIDOS-CM (S2009TIC-1465) and GPD-UCM (UCM-BSCH-GR58/ 08910502).
} 
López-Fraguas et al.

$\begin{array}{ll}\text { coin } \rightarrow 0 & \text { repeat }(X) \rightarrow X: \text { repeat }(X) \\ \text { coin } \rightarrow 1 & \text { heads }(X: Y: Y s) \rightarrow(X, Y)\end{array}$

Fig. 1. A non-terminating and non-confluent program

theory of TRS underlies many of the proposals made in the last decades for socalled functional logic programming, attempting to integrate into a single language the main features of both functional and logic programming - see (DeGroot and Lindstrom 1986, Hanus 1994, Hanus 2007) for surveys corresponding to different historical stages of the development of functional logic languages- Typically, functional logic programs are modeled by some kind of TRS to define functions, and logic programming capabilities are achieved by using some kind of narrowing as operational mechanism. Narrowing, a notion coming from the field of automated theorem proving, generalizes rewriting by using unification instead of matching in reduction steps. Up to 14 different variants of narrowing were identified in (Hanus 1994) as being used in different proposals for the integration of functional and logic programming.

Modern functional logic languages like Curry (Hanus et al. 1995; Hanus (ed.) 2006) or Toy (López-Fraguas and Sánchez-Hernández 1999 Caballero and Sánchez 2006) consider that programs are constructor-based term rewrite systems, possibly non-terminating and non-confluent, thus defining possibly non-strict nondeterministic functions. For instance, in the program of Figure 1 non-confluence comes from the two rules of coin and non-termination is due to the rule for repeat.

For non-determinism, those systems adopt call-time choice semantics (Hussmann 1993: González-Moreno et al. 1999), also called sometimes singular semantics (Søndergaard and Sestoft 1992). Loosely speaking, call-time choice means to pick a value for each argument of a function application before applying it. Call-time choice is easier to understand and implement in combination with strict semantics and eager evaluation in terminating systems as in (Hussmann 1993), but can be made also compatible - via partial values and sharing - with non-strictness and laziness in the presence of non-termination.

In the example of Figure 1 the expression heads(repeat(coin)) can take, under call-time choice, the values $(0,0)$ and $(1,1)$, but not $(0,1)$ or $(1,0)$. The example illustrates also a key point here: ordinary term rewriting (called run-time choice in (Hussmann 1993)) is an unsound procedure for call-time choice semantics, since a possible term rewriting derivation is:

$$
\begin{aligned}
& \text { heads }(\text { repeat }(\operatorname{coin})) \rightarrow \text { heads }(\operatorname{coin}: \operatorname{repeat}(\operatorname{coin})) \rightarrow \\
& \text { heads }(0: \operatorname{repeat}(\operatorname{coin})) \rightarrow \text { heads }(0: \operatorname{coin}: \operatorname{repeat}(\operatorname{coin})) \rightarrow \\
& \text { heads }(0: 1: \operatorname{repeat}(\operatorname{coin})) \rightarrow(0,1)
\end{aligned}
$$

In operational terms, call-time choice requires to share the value of all copies of a given subexpression created during reduction (all the occurrences of coin, in the reduction above). In contrast, with ordinary term rewriting all copies evolve independently.

It is commonly accepted (see e.g. (Hanus 2007)) that call-time choice semantics 
combined with non-strict semantics is adequately formally expressed by the CRWL framework 1 (González-Moreno et al. 1996: González-Moreno et al. 1999), whose main component is a proof calculus that determines the semantics of programs and expressions. The flexibility and usefulness of CRWL is evidenced by the large set of extensions that have been devised for it, to cope with relevant aspects of declarative programming: higher order functions, types, constraints, constructive failure, ...; see (Rodríguez-Artalejo 2001) for a survey on the CRWL approach. However, a drawback of the CRWL-framework is its lack of a proper one-step reduction mechanism that could play a role similar to term rewriting with respect to equational logic. Certainly CRWL includes operational procedures in the form of goal-solving calculi (González-Moreno et al. 1999 Vado-Vírseda 2003) to solve so-called joinability conditions, but they are too complex to be seen as a basic way to explain or understand how a reduction can proceed in the presence of non-strict non-deterministic functions with call-time choice semantics.

On the other hand, other works have been more influential on the operational side of the field, specially those based on the notion of needed narrowing Antoy et al. 1994 Antoy et al. 2000), a variant of narrowing that organizes the evaluation of arguments in function calls in an adequate way (optimal, for some classes of programs). Needed narrowing became the 'official' operational procedure of functional logic languages, and has also been subject of several variations and improvements (see (Hanus 2007, Escobar et al. 2005)).

These two coexisting branches of research (one based on CRWL, and the other based on classical term rewriting, mostly via needed narrowing) have remained disconnected for many years from the technical point of view, despite the fact that they both refer to what intuitively is the same programming language paradigm.

A major problem to establish the connection was that the theory underlying needed narrowing is classical term rewriting, which, as we saw above, is not valid for non-determinism with call-time choice semantics. This was not a flaw in the conception of needed narrowing, as it emerged in a time when non-deterministic functions had not yet started to play a distinctive role in the functional logic programming paradigm. The problem is overcome in practice by adding a sharing mechanism to the implementation of narrowing, using for instance standard Prolog programming techniques (Cheong and Fribourg 1993, Loogen et al. 1993, Antoy and Hanus 2000). But this is merely an implementation patch that cannot be used as a precise and sound technical basis for the application of results and techniques from the semantic side to the operational side and vice versa. Other works, specially (Echahed and Janodet 1998; Albert et al. 2005) have addressed in a more formal way the issue of sharing in functional logic programming, but they are not good starting points to establish a relationship with the CRWL world (see 'Related work' below).

In (López-Fraguas et al. 2007b) we aimed at establishing a bridge, by looking for

\footnotetext{
${ }^{1}$ CRWL stands for Constructor Based ReWriting Logic.
} 
a new variant of term rewriting tailored to call-time choice as realized by CRWL, trying to fulfil the following requirements:

- it should be based on a notion of rewrite step useful to follow how a computation proceeds step by step.

- it should be simple enough to be easily understandable for non-expert potential users. (e.g., students or novice programmers) of functional logic languages adopting call-time choice.

- it should be provably equivalent to CRWL, as a well-established technical formulation of call-time choice.

- it should serve as a basis of subsequent notion of narrowing and evaluation strategies.

That was realized in (López-Fraguas et al. 2007b) by means of let-rewriting, a simple modification of term rewriting using local bindings in the form of letexpressions to express sharing. Let-rewriting will be fully presented in Section 4 but its main intuitions can be summarized as follows:

(i) do not rewrite a function call if any of its arguments is evaluable (i.e., still contains other function calls), even if there is a matching rule;

(ii) instead, extract those evaluable arguments to outer let-bindings of the form let $X=e$ in $e^{\prime}$;

(iii) if after some reduction steps the definiens $e$ of the let-binding becomes a constructor term $t$ - a value - then the binding $X / t$ can be made effective in the body $e^{\prime}$. In this way, the values obtained for $e$ in the reduction are shared, and therefore call-time choice is respected.

Consider, for instance, the program example of Figure 1 and the expression

$$
\text { heads(repeat(coin)) }
$$

for which we previously performed an ordinary term rewriting reduction ending in $(0,1)$. Now we are going to apply liberally the previous intuitive hints as a first illustration of let-rewriting. Note first that no rewrite step using a program rule can be done with the whole expression heads(repeat(coin)), since in this case there is no matching rule. But we can extract the argument repeat(coin) to a let-binding, obtaining:

$$
\text { let } X=\text { repeat(coin) in heads }(X)
$$

Now we cannot rewrite repeat(coin), even though the program rule for repeat matches it, because coin is evaluable. Again, we can create a let-binding for coin, that will be used to share the value selected for coin, if at any later step in the reduction coin is indeed reduced:

$$
\text { let } Y=\text { coin in let } X=\operatorname{repeat}(Y) \text { in heads }(X)
$$

At this point there is no problem with rewriting repeat $(Y)$, which gives:

$$
\text { let } Y=\text { coin in let } X=Y: \operatorname{repeat}(Y) \text { in heads }(X)
$$


Rewriting repeat $(Y)$ again, we have:

$$
\text { let } Y=\text { coin in let } X=Y: Y: \operatorname{repeat}(Y) \text { in heads }(X)
$$

Reducing repeat $(Y)$ indefinitely leads to non-termination, but, at the same time, its presence inhibits the application of the binding for $X$. What we can do is creating a new let-binding for the remaining repeat $(Y)$, which results in:

$$
\text { let } Y=\text { coin in let } Z=\operatorname{repeat}(Y) \text { in let } X=Y: Y: Z \text { in heads }(X)
$$

Now, the binding for $X$ can be performed, obtaining:

$$
\text { let } Y=\text { coin in let } Z=\operatorname{repeat}(Y) \text { in heads }(Y: Y: Z)
$$

At this point, we can use the rule for heads to evaluate heads $(Y: Y: Z)$, because nothing evaluable remains in its argument $Y: Y: Z$, arriving at:

$$
\text { let } Y=\text { coin in let } Z=\operatorname{repeat}(Y) \text { in }(Y, Y)
$$

We proceed now by reducing coin, for instance, to 0 (reducing it to 1 is also possible):

$$
\text { let } Y=0 \text { in let } Z=\operatorname{repeat}(Y) \text { in }(Y, Y)
$$

Performing the binding for $Y$ leads to:

$$
\text { let } Z=\operatorname{repeat}(0) \text { in }(0,0)
$$

Since $Z$ does not occur in $(0,0)$, its binding is junk that could be deleted (there will be a rule for that in the definition of let-rewriting), and the reduction is finished yielding the value

It is apparent that $(1,1)$ is another possible result, but not $(0,1)$ nor $(1,0)$, a behavior coherent with call-time choice.

In this example we have tried to proceed in a more or less natural 'lazy' way. However, the previous intuitive precepts - and its complete and precise realization in Section 4 do not assume any particular strategy for organizing reductions, but only determine which are the 'legal movements' in call-time choice respectful reductions. Strategies have been left aside in the paper, not only for simplicity, but also to keep them independent of the basic rules for term rewriting with sharing (see however Section 6.2).

Let-rewriting was later on extended to cope with narrowing (López-Fraguas et al. 2009c) and higher order features (López-Fraguas et al. 2008).

This paper is a substantially revised and completed presentation of the theory of first order let-rewriting and let-narrowing proposed in (López-Fraguas et al. 2007b; López-Fraguas et al. 2009c); some contents have been also taken from (LópezFraguas et al. 2008). Here, we unify technically those papers and develop a deeper investigation of the properties of let-rewriting and related semantics issues.

Related work Our let-rewriting and let-narrowing relations are not the only nor the first formal operational procedures tuned up to accomplish with the calltime choice semantics of functional logic languages. We have already mentioned the 
goal-solving calculi associated to the CRWL-framework and its variants (GonzálezMoreno et al. 1999; González-Moreno et al. 1997; Vado-Vírseda 2003).

A natural option to express different levels of sharing in rewriting is given by the theory of term graph rewriting (Barendregt et al. 1987; Plump 2001). In (Echahed and Janodet 1997, Echahed and Janodet 1998), the theory of needed rewriting and narrowing was extended to the framework of so-called admissible graph rewriting systems, aiming at formally modeling the operational behavior of functional logic programs. Originally, those works considered orthogonal systems, and extra variables were not allowed. These restrictions were dropped in (Antoy et al. 2007) (however, a formal treatment of the extension is missing).

As a matter of fact, our let-rewriting relation can be understood as a particular textual adaptation and presentation of term graph rewriting in which a shared node is made explicit in the syntax by giving it a name in a let-binding. The achievements of Echahed's works are somehow incomparable to ours, even if both are attempts to formalize sharing in constructor based systems. They focus and succeed on adapting known optimal strategies to the graph rewriting and narrowing setting; they also take profit of the fine-grained descriptions permitted by graphs to manage aspects of data structures like cycles or pointers. However, they do not try to establish a technical relationship with other formulations of call-time choice. In contrast, proving equivalence of our operational formalisms wrt. the CRWL semantic framework has been a main motivation of our work, but we do not deal with the issue of strategies, except for a short informal discussion at the end of the paper.

It is our thought that proving equivalence with respect to CRWL of term graph rewriting as given in (Echahed and Janodet 1997) would have been a task much harder than the route we follow here. We see a reason for it. The basic pieces that term rewriting and CRWL work with are purely syntactic: terms, substitutions, etc. Graph rewriting recast these notions in terms of graphs, homomorphisms, etc. In contrast, let-rewriting and let-narrowing keep the same set of basic pieces of term rewriting and CRWL. In this way, the formalisms are relatively close and moving from one to another becomes technically more natural and comfortable. This applies also to some further developments of our setting that we have made so far, like the extension to higher order features given in (López-Fraguas et al. 2008), the combination of semantics proposed in (López-Fraguas et al. 2009a), or the application of let-rewriting as underlying formal notion of reduction for type systems in functional logic languages (López-Fraguas et al. 2010b; López-Fraguas et al. 2010a).

Another proposal that can be seen as reformulation of graph rewriting was given in (Albert et al. 2005), inspired in Launchbury's natural semantics (Launchbury 1993) for lazy evaluation in functional programming. It presents two operational (natural and small-step) semantics for functional logic programs supporting sharing and residuation (a specific feature of Curry). These semantics use a flat representation of programs coming from an implicit program transformation encoding the demand analysis used by needed narrowing, and some kind of heaps to express bindings for variables. As in our case, let-expressions are used to express sharing. 
The approach is useful as a technical basis for implementation and program manipulation purposes; but we think that the approach is too low-level and close to a particular operational strategy to be a completely satisfactory choice as basic abstract reduction mechanism for call-time choice. In (López-Fraguas et al. 2007a) we established a technical relation of CRWL with the operational procedures of (Albert et al. 2005). But this turned out to be a really hard task, even if it was done only for a restricted class of programs and expressions.

Our work focuses on term rewriting systems as basic formalism, as happens with the majority of papers about the foundations of functional logic programming, in particular the CRWL-series. The idea of reformulating graph rewriting in a syntactic style by expressing sharing through let-bindings has been applied also to other contexts, most remarkably to $\lambda$-calculus considered as a basis of functional programming (Ariola and Arvind 1995; Ariola et al. 1995, Ariola and Felleisen 1997; Maraist et al. 1998). In a different direction, but still in relation with $\lambda$-calculus, other papers (Kutzner and Schmidt-Schauß 1998, Schmidt-Schauß and Machkasova 2008) have extended it with some kind of non-deterministic choice, an idea that comes back to McCarthy's amb (McCarthy 1963). As a final note, we should mention that our initial ideas about let-rewriting were somehow inspired by (López-Fraguas and Sánchez-Hernández 2001; Sánchez-Hernández 2004) where indexed unions of set expressions - a construction generalizing the idea of let-expressions - were used to express sharing in an extension of CRWL to deal with constructive failure.

The rest of the paper is organized as follows. Section 2 presents some preliminaries about term rewriting and the CRWL framework; although with them the paper becomes almost self-contained, some familiarity with the basic notions of TRS certainly help to read the paper. Section 3 contains a first discussion about failed or partial solutions to the problem of expressing non-strict call-time choice by a simple notion of rewriting. Section 4 is the central part of the paper. First, it introduces local bindings in the syntax to express sharing, defines let-rewriting as an adequate notion of rewriting for them and proves some intrinsic properties of let-rewriting. After that, in Section 4.2, we extend the CRWL-logic to a new $\mathrm{CRWL}_{l e t}$-logic able to deal with lets in programs and expressions, and we investigate in depth the properties of the induced semantics, mostly through the notion of hypersemantics. Finally, in Section 4.3 we prove results of soundness and completeness of let-rewriting with respect to $\mathrm{CRWL}_{l e t}$, which have as corollary the equivalence of both, and hence the equivalence of let-rewriting and CRWL for programs and expressions not containing lets, as the original CRWL ones are. Section 5 aims at showing the power of having reduction and semantics as equivalent interchangeable tools for reasoning, including a remarkable case study. In Section 6 we generalize the notion of let-rewriting to that of let-narrowing and give soundness and completeness results of the latter with respect to the former. At the end of the section we give some hints on how computations can be organized according to known narrowing strategies. Section 7 addresses the relationship between letrewriting and classical term rewriting, proving in particular their equivalence for semantically deterministic programs. Finally, Section 8 analyzes our contribution 
and suggests further work. For the sake of readability, most of the (fully detailed) proofs have been moved to the online appendix of the paper.

\section{Preliminaries}

\subsection{Constructor based term rewriting systems}

We assume a fixed first order signature $\Sigma=C S \cup F S$, where $C S$ and $F S$ are two disjoint sets of constructor and defined function symbols respectively, each of them with an associated arity. We write $C S^{n}$ and $F S^{n}$ for the set of constructor and function symbols of arity $n$ respectively, and $\Sigma^{n}$ for $C S^{n} \cup F S^{n}$. As usual notations we write $c, d, \ldots$ for constructors, $f, g, \ldots$ for functions and $X, Y, \ldots$ for variables taken from a denumerable set $\mathcal{V}$. The notation $\bar{o}$ stands for tuples of any kind of syntactic objects.

The set Exp of expressions is defined as Exp $\ni e::=X \mid h\left(e_{1}, \ldots, e_{n}\right)$, where $X \in$ $\mathcal{V}, h \in \Sigma^{n}$ and $e_{1}, \ldots, e_{n} \in E x p$. The set CTerm of constructed terms (or c-terms) has the same definition of Exp, but with $h$ restricted to $C S^{n}$ (so CTerm $\varsubsetneqq E x p$ ). The intended meaning is that Exp stands for evaluable expressions, i.e., expressions that can contain (user-defined) function symbols, while CTerm stands for data terms representing values. We will write $e, e^{\prime}, \ldots$ for expressions and $t, s, p, t^{\prime}, \ldots$ for c-terms. The set of variables occurring in an expression $e$ will be denoted as $\operatorname{var}(e)$.

Contexts (with one hole) are defined by Cntxt $\ni \mathcal{C}::=[] \mid h\left(e_{1}, \ldots, \mathcal{C}, \ldots, e_{n}\right)$, where $h \in \Sigma^{n}$. The application of a context $\mathcal{C}$ to an expression $e$, written as $\mathcal{C}[e]$, is defined inductively as follows:

$$
\begin{aligned}
{[][e] } & =e \\
h\left(e_{1}, \ldots, \mathcal{C}, \ldots, e_{n}\right)[e] & =h\left(e_{1}, \ldots, \mathcal{C}[e], \ldots, e_{n}\right)
\end{aligned}
$$

Substitutions are finite mappings $\sigma: \mathcal{V} \longrightarrow$ Exp which extend naturally to $\sigma$ : $E x p \longrightarrow E x p$. We write $e \sigma$ for the application of the substitution $\sigma$ to $e$. The domain and variable range of a substitution $\sigma$ are defined as $\operatorname{dom}(\sigma)=\{X \in \mathcal{V} \mid$ $X \sigma \neq X\}$ and $\operatorname{vran}(\sigma)=\bigcup_{X \in \operatorname{dom}(\sigma)} \operatorname{var}(X \sigma)$. By $\left[X_{1} / e_{1}, \ldots, X_{n} / e_{n}\right]$ we denote the substitution $\sigma$ such that $Y \sigma=e_{i}$ if $Y \equiv X_{i}$ for some $X_{i} \in\left\{X_{1}, \ldots, X_{n}\right\}$, and $Y \sigma=Y$ otherwise. Given a set of variables $D$, the notation $\left.\sigma\right|_{D}$ represents the substitution $\sigma$ restricted to $D$ and $\left.\sigma\right|_{\backslash D}$ is a shortcut for $\left.\sigma\right|_{(\mathcal{V} \backslash D)}$. A c-substitution is a substitution $\theta$ such that $X \theta \in C T$ erm for all $X \in \operatorname{dom}(\theta)$. We write $S u b s t$ and CSubst for the sets of substitutions and c-substitutions respectively.

A term rewriting system is any set of rewrite rules of the form $l \rightarrow r$ where $l, r \in \operatorname{Exp}$ and $l \notin \mathcal{V}$. A constructor based rewrite rule or program rule has the form $f\left(p_{1}, \ldots, p_{n}\right) \rightarrow r$ where $f \in F S^{n}, r \in \operatorname{Exp}$ and $\left(p_{1}, \ldots, p_{n}\right)$ is a linear tuple of c-terms, where linear means that no variable occurs twice in the tuple. Notice that we allow $r$ to have extra variables (i.e., variables not occurring in the lefthand side). To be precise, we say that $X$ is an extra variable in the rule $l \rightarrow r$ iff $X \in \operatorname{var}(r) \backslash \operatorname{var}(l)$, and by $v \operatorname{Extra}(R)$ we denote the set of extra variables in a rule $R$. Then a constructor system or program $\mathcal{P}$ is any set of program rules, i.e., a term rewriting system composed only of program rules.

Given a program $\mathcal{P}$, its associated rewrite relation $\rightarrow_{\mathcal{P}}$ is defined as $\mathcal{C}[l \sigma] \rightarrow_{\mathcal{P}}$ 
$\mathcal{C}[r \sigma]$ for any context $\mathcal{C}$, rule $l \rightarrow r \in \mathcal{P}$ and $\sigma \in$ Subst. There, the subexpression $l \sigma$ is called the redex used in that rewriting step. Notice that $\sigma$ can instantiate extra variables to any expression. For any binary relation $\mathcal{R}$ we write $\mathcal{R}^{*}$ for the reflexive and transitive closure of $\mathcal{R}$, and $\mathcal{R}^{n}$ for the composition of $\mathcal{R}$ with itself $n$ times. We write $e_{1} \stackrel{*}{\rightarrow} \mathcal{p} e_{2}$ for a term rewriting derivation or reduction from $e_{1}$ to $e_{2}$, and $e_{1} \stackrel{n}{\rightarrow} e_{2}$ for a $n$-step reduction. $e_{2}$ is a normal form wrt. $\rightarrow_{\mathcal{P}}$, written as $\downarrow^{\mathcal{P}} e_{2}$, if there is not any $e_{3}$ such that $e_{2} \rightarrow_{\mathcal{P}} e_{3}$; and $e_{2}$ is a normal form for $e_{1}$ wrt. $\rightarrow \mathcal{P}$, written as $e_{1} \downarrow^{\mathcal{P}} e_{2}$, iff $e_{1} \stackrel{*}{\rightarrow} \mathcal{P} e_{2}$ and $e_{2}$ is a normal form. When presenting derivations, we will sometimes underline the redex used at each rewriting step. In the following, we will usually omit the reference to $\mathcal{P}$ when writing $e_{1} \rightarrow \mathcal{P} e_{2}$, or denote it by $\mathcal{P} \vdash e_{1} \rightarrow e_{2}$.

A program $\mathcal{P}$ is confluent if for any $e, e_{1}, e_{2} \in \operatorname{Exp}$ such that $e \rightarrow_{\mathcal{P}}^{*} e_{1}, e \rightarrow_{\mathcal{P}}^{*} e_{2}$ there exists $e_{3} \in \operatorname{Exp}$ such that both $e_{1} \rightarrow_{\mathcal{P}}^{*} e_{3}$ and $e_{2} \rightarrow_{\mathcal{P}}^{*} e_{3}$.

\subsection{The CRWL framework}

We present here a simplified version of the CRWL framework (González-Moreno et al. 1996: González-Moreno et al. 1999). The original CRWL logic considered also the possible presence of joinability constraints as conditions in rules in order to give a better treatment of strict equality as a built-in, a subject orthogonal to the aims of this work. Furthermore, it is possible to replace conditions by the use of an if_then function, as has been technically proved in (Sánchez-Hernández 2004) for CRWL and in (Antoy 2005) for term rewriting. Therefore, we consider only unconditional program rules.

In order to deal with non-strictness at the semantic level, we enlarge $\Sigma$ with a new constant (i.e., a 0-ary constructor symbol) $\perp$ that stands for the undefined value. The sets $\operatorname{Exp}_{\perp}, C T e r m_{\perp}, S u b s t_{\perp}, C S u b s t_{\perp}$ of partial expressions, etc., are defined naturally. Notice that $\perp$ does not appear in programs. Partial expressions are ordered by the approximation ordering $\sqsubseteq$ defined as the least partial ordering satisfying $\perp \sqsubseteq e$ and $e \sqsubseteq e^{\prime} \Rightarrow \mathcal{C}[e] \sqsubseteq \mathcal{C}\left[e^{\prime}\right]$ for all $e, e^{\prime} \in \operatorname{Exp}_{\perp}, \mathcal{C} \in$ Cntxt. This partial ordering can be extended to substitutions: given $\theta, \sigma \in S u b s t_{\perp}$ we say $\theta \sqsubseteq \sigma$ if $X \theta \sqsubseteq X \sigma$ for all $X \in \mathcal{V}$.

The semantics of a program $\mathcal{P}$ is determined in CRWL by means of a proof calculus able to derive reduction statements of the form $e \rightarrow t$, with $e \in \operatorname{Exp}_{\perp}$ and $t \in C T e r m_{\perp}$, meaning informally that $t$ is (or approximates to) a possible value of $e$, obtained by evaluating $e$ using $\mathcal{P}$ under call-time choice.

The CRWL-proof calculus is presented in Figure 2 Rule (B) allows any expression to be undefined or not evaluated (non-strict semantics). Rule (OR) expresses that to evaluate a function call we must choose a compatible program rule, perform parameter passing (by means of a c-substitution $\theta$ ) and then reduce the right-hand side. The use of c-substitutions in (OR) is essential to express call-time choice; notice also that by the effect of $\theta$ in (OR), extra variables in the right-hand side of a rule can be replaced by any partial c-term, but not by any expression as in ordinary term rewriting $\rightarrow_{\mathcal{P}}$. We write $\mathcal{P} \vdash_{C R W L} e \rightarrow t$ to express that $e \rightarrow t$ is derivable in 


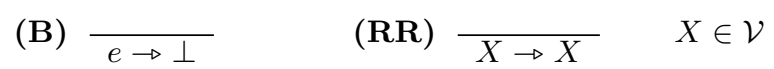

$$
\begin{aligned}
& \text { (DC) } \frac{e_{1} \rightarrow t_{1} \ldots e_{n} \rightarrow t_{n}}{c\left(e_{1}, \ldots, e_{n}\right) \rightarrow c\left(t_{1}, \ldots, t_{n}\right)} \quad c \in C S^{n} \\
& \text { (OR) } \frac{e_{1} \rightarrow p_{1} \theta \ldots e_{n} \rightarrow p_{n} \theta r \theta \rightarrow t}{f\left(e_{1}, \ldots, e_{n}\right) \rightarrow t} \quad \begin{array}{l}
\left(f\left(p_{1}, \ldots, p_{n}\right) \rightarrow r\right) \in \mathcal{P} \\
\theta \in C \text { Subst } \\
\perp
\end{array}
\end{aligned}
$$

Fig. 2. Rules of CRWL

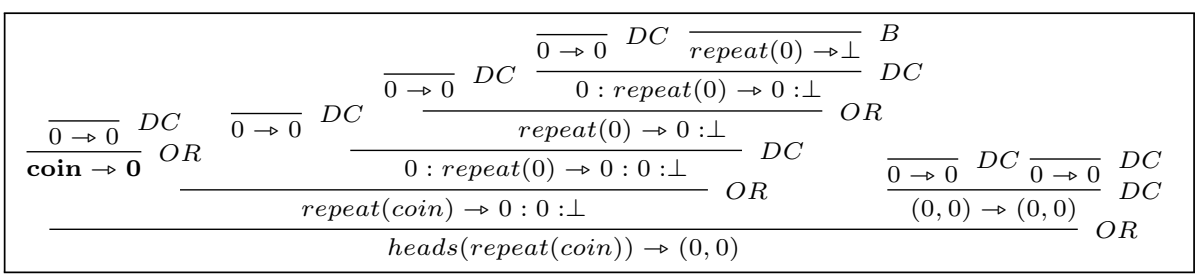

Fig. 3. A CRWL-derivation for heads $($ repeat $($ coin $)) \rightarrow(0,0)$

the CRWL-calculus using the program $\mathcal{P}$, but in many occasions we will omit the mention to $\mathcal{P}$, writing simply $e \rightarrow t$.

\section{Definition 1 (CRWL-denotation)}

Given a program $\mathcal{P}$, the $C R W L$-denotation of an expression $e \in E x p_{\perp}$ is defined as

$$
\llbracket e \rrbracket_{C R W L}^{\mathcal{P}}=\left\{t \in C T e r m_{\perp} \mid \mathcal{P} \vdash_{C R W L} e \rightarrow t\right\}
$$

We will usually omit the subscript CRWL and/or the superscript $\mathcal{P}$ when implied by the context.

As an example, Figure 3 shows a $C R W L$-derivation or $C R W L$-proof for the statement heads(repeat $(\operatorname{coin})) \rightarrow(0,0)$, using the program of Figure 1 . Observe that in the derivation there is only one reduction statement for coin (namely coin $\rightarrow 0$ ), and the obtained value 0 is then shared in the whole derivation, as corresponds to call-time choice.

In alternative derivations, coin could be reduced to 1 (or to $\perp$ ). It is easy to check that:

$$
\llbracket \text { heads }(\text { repeat }(\operatorname{coin})) \rrbracket=\{(0,0),(1,1),(\perp, 0),(0, \perp),(\perp, 1),(1, \perp),(\perp, \perp), \perp\}
$$

Note that $(1,0),(0,1) \notin \llbracket$ heads $($ repeat $($ coin $)) \rrbracket$.

We stress the fact that the CRWL-calculus is not an operational mechanism for executing programs, but a way of describing the logic of programs. In particular, the rule (B) is a semantic artifact to reflect in a CRWL-proof of a statement $e \rightarrow t$ the fact that, for obtaining $t$ as value of $e$, one does not need to know the value of a certain subexpression $e^{\prime}$ (to which the rule (B) is applied). But the calculus comes with no indication of when to apply (B) in a successful proof. At the operational 
level, the CRWL framework is accompanied with various lazy narrowing-based goalsolving calculi (González-Moreno et al. 1999, Vado-Vírseda 2003) not considered in this paper.

One of the most important properties of CRWL is its compositionality, a property very close to the DET-additivity property for algebraic specifications of (Hussmann 1993 ) or the referential transparency property of (Sondergaard and Sestoft 1990). Compositionality shows that the CRWL-denotation of any expression placed in a context only depends on the CRWL-denotation of that expression. This implies that the semantics of a whole expression depends only on the semantics of its constituents, as shown by the next result, which is an adaptation of a similar one proved for the higher order case in (López-Fraguas et al. 2008).

Theorem 1 (Compositionality of CRWL)

For any $\mathcal{C} \in C n t x t, e, e^{\prime} \in \operatorname{Exp}_{\perp}$

$$
\llbracket \mathcal{C}[e] \rrbracket=\bigcup_{t \in \llbracket e \rrbracket} \llbracket \mathcal{C}[t] \rrbracket
$$

As a consequence: $\llbracket e \rrbracket=\llbracket e^{\prime} \rrbracket \Leftrightarrow \forall \mathcal{C} \in C n t x t . \llbracket \mathcal{C}[e] \rrbracket=\llbracket \mathcal{C}\left[e^{\prime}\right] \rrbracket$

According to this result we can express for example

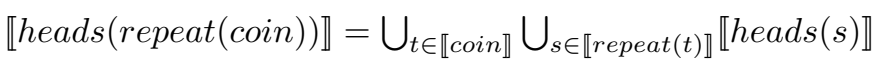

The right hand side has an intuitive reading that reflects call-time choice: get a value $t$ of coin, then get a value $s$ of repeat $(t)$ and then get a value of heads $(s)$.

In Theorem 2 we give an alternative formulation to the compositionality property. Although it is essentially equivalent to Theorem 1, it is a somehow more abstract statement, based on the notion of denotation of a context introduced in Definition 2. Our main reason for developing such alternative is to give good insights for the compositionality results of the extension of CRWL to be presented in Section 4.3 .

We will use sometimes $D e n$ as an alias for $\mathcal{P}\left(C T e r m_{\perp}\right)$, i.e, for the kind of objects that are CRWL-denotations of expressions ${ }^{2}$. We define the denotation of a context $\mathcal{C}$ as a denotation transformer that reflects call-time choice.

Definition 2 (Denotation of a context)

Given $\mathcal{C} \in C$ ntxt, its denotation is a function $\llbracket \mathcal{C} \rrbracket:$ Den $\rightarrow$ Den defined as

$$
\llbracket \mathcal{C} \rrbracket \delta=\bigcup_{t \in \delta} \llbracket \mathcal{C}[t] \rrbracket, \forall \delta \in D e n
$$

With this notion, compositionality can be trivially re-stated as follows:

\footnotetext{
${ }^{2}$ Den is indeed a superset of the set of actual denotations, which are particular elements of $\mathcal{P}\left(\right.$ CTerm $\left._{\perp}\right)$, namely cones — see (González-Moreno et al. 1999- But this is not relevant to the use we make of Den.
} 
Theorem 2 (Compositionality of CRWL, version 2)

For any $\mathcal{C} \in$ Cntxt and $e, e^{\prime} \in \operatorname{Exp}_{\perp}$

$$
\llbracket \mathcal{C}[e] \rrbracket=\llbracket \mathcal{C} \rrbracket \llbracket e \rrbracket
$$

As a consequence: $\llbracket e \rrbracket=\llbracket e^{\prime} \rrbracket \Leftrightarrow \forall \mathcal{C} \in C n t x t . \llbracket \mathcal{C}[e] \rrbracket=\llbracket \mathcal{C}\left[e^{\prime}\right] \rrbracket$

The formulation of compositionality given by Theorem 2 makes even more apparent than Theorem 1 the fact that the syntactic decomposition of an expression $e$ in the form $\mathcal{C}\left[e^{\prime}\right]$ has a direct semantic counterpart, in the sense that the semantics of $e$ is determined by the semantics of its syntactic constituents $\mathcal{C}$ and $e^{\prime}$. However, Theorems 1 and 2 are indeed of the same strength, since each of them can be easily proved from the other.

\section{CRWL and rewriting: a first discussion}

Before presenting let-rewriting we find interesting to discuss a couple of (in principle) shorter solutions to the problem of expressing non-strict call-time choice semantics by means of a simple one-step reduction relation. A first question is whether a new relation is needed at all: maybe call-time choice can be expressed by ordinary term rewriting via a suitable program transformation. The next result shows that in a certain technical sense this is not possible: due to different closedness under substitution and compositionality properties of call-time choice and term rewriting, none of them can be naturally simulated by each other.

Proposition 1

There is a program $\mathcal{P}$ for which the following two conditions hold:

i) no term rewriting system (constructor based or not) $\mathcal{P}^{\prime}$ verifies

$$
\mathcal{P} \vdash_{C R W L} e \rightarrow t \text { iff } \mathcal{P}^{\prime} \vdash e \rightarrow^{*} t \text {, for all } e \in \operatorname{Exp}, t \in C T e r m
$$

ii) no program $\mathcal{P}^{\prime}$ verifies

$$
\mathcal{P} \vdash e \rightarrow^{*} t \text { iff } \mathcal{P}^{\prime} \vdash_{C R W L} e \rightarrow t, \text { for all } e \in \operatorname{Exp}, t \in C T e r m
$$

Proof

The following simple program $\mathcal{P}$ suffices:

$$
f(X) \rightarrow c(X, X) \quad \text { coin } \rightarrow 0 \quad \text { coin } \rightarrow 1
$$

i) We reason by contradiction. Assume there is a term rewriting system $\mathcal{P}^{\prime}$ such that: $\mathcal{P} \vdash_{C R W L} e \rightarrow t \Leftrightarrow e \rightarrow_{\mathcal{P}^{\prime}}^{*} t$, for all $e, t$. Since $\mathcal{P} \vdash_{C R W L} f(X) \rightarrow$ $c(X, X)$, we must have $f(X) \rightarrow_{\mathcal{P}^{\prime}}^{*} c(X, X)$. Now, since $\rightarrow_{\mathcal{P}^{\prime}}^{*}$ is closed under substitutions (Baader and Nipkow 1998), we have $f($ coin $) \rightarrow_{\mathcal{P}^{\prime}}^{*} c($ coin, coin), and therefore $f(\operatorname{coin}) \rightarrow{ }_{\mathcal{P}^{\prime}}^{*} c($ coin, coin $) \rightarrow_{\mathcal{P}^{\prime}}^{*} c(0,1)$. But it is easy to see that $\mathcal{P} \vdash_{C R W L}$ $f(\operatorname{coin}) \rightarrow c(0,1)$ does not hold.

ii) Assume now there is a program $\mathcal{P}^{\prime}$ such that: $\mathcal{P} \vdash e \rightarrow^{*} t \Leftrightarrow \mathcal{P}^{\prime} \vdash_{C R W L} e \rightarrow t$, for all $e, t$. Since $\mathcal{P} \vdash f(\operatorname{coin}) \rightarrow^{*} c(0,1)$, we have $\mathcal{P}^{\prime} \vdash_{C R W L} f(\operatorname{coin}) \rightarrow c(0,1)$. By compositionality of call-time choice (Theorem 1 , there must exist a possibly partial 


$$
\begin{aligned}
& \left(\mathbf{B}^{r w}\right) \quad \mathcal{C}[e] \quad \longmapsto \mathcal{C}[\perp] \quad \forall \mathcal{C} \in \text { Cntxt, } e \in \operatorname{Exp}_{\perp} \\
& \left(\mathbf{O R}^{r w}\right) \quad \mathcal{C}\left[f\left(t_{1} \theta, \ldots, t_{n} \theta\right)\right] \quad \longmapsto \mathcal{C}[r \theta] \quad \forall \mathcal{C} \in C n t x t, f\left(t_{1}, \ldots, t_{n}\right) \rightarrow r \in \mathcal{P}, \\
& \theta \in C S u b s t_{\perp}
\end{aligned}
$$

Fig. 4. A one-step reduction relation for non-strict call-time choice

$t \in C T e r m_{\perp}$ such that $\mathcal{P}^{\prime} \vdash_{C R W L}$ coin $\rightarrow t$ and $\mathcal{P}^{\prime} \vdash_{C R W L} f(t) \rightarrow c(0,1)$. Now we distinguish cases on the value of $t$ :

(a) If $t \equiv \perp$, then monotonicity of $C R W L$-derivability - see González-Moreno et al. 1999) or Proposition 3 below - proves that $\mathcal{P}^{\prime} \vdash_{C R W L} f(s) \rightarrow c(0,1)$ for any $s \in C$ Term $_{\perp}$, in particular $\mathcal{P}^{\prime} \vdash_{C R W L} f(0) \rightarrow c(0,1)$. Then, by the assumption on $\mathcal{P}^{\prime}$, it should be $\mathcal{P} \vdash f(0) \rightarrow^{*} c(0,1)$, but this is not true.

(b) If $t \equiv 0$, then $\mathcal{P}^{\prime} \vdash f(0) \rightarrow c(0,1)$ as before. The cases $t \equiv 1, t \equiv Y$ or $t \equiv d(\bar{s})$ for a constructor $d$ different from 0,1 lead to similar contradictions.

Notice that Proposition 1 does not make any assumption about signatures: in any of $i$ ) or ii), no extension of the signature can lead to a simulating $\mathcal{P}^{\prime}$. This does not contradict Turing completeness of term rewriting systems. Turing completeness arguments typically rely on encodings not preserving the structure of data, something not contemplated in Proposition 1 .

In a second trial, requiring minimal changes over ordinary term rewriting, we impose that the substitution $\theta$ in a rewriting step must be a c-substitution, as in the rule (OR) of CRWL. This is done in the one-step rule $\left(\mathbf{O R}^{r w}\right)$ in Figure 4 According to it, the step heads(repeat $($ coin $)) \rightarrow$ heads $($ coin $:$ repeat $($ coin $))$ in the introductory example of Figure 1 would not be legal anymore. However, $\left(\mathrm{OR}^{r w}\right)$ corresponds essentially to innermost evaluation, and is not enough to deal with non-strictness, as the following example shows:

\section{Example 1}

Consider the rules $f(X) \rightarrow 0$ and loop $\rightarrow$ loop. With a non-strict semantics $f($ loop $)$ should be reducible to 0 . But $\left(\mathrm{OR}^{r w}\right)$ does not allow the step $f(l o o p) \rightarrow 0$; only $f($ loop $) \rightarrow f($ loop $) \rightarrow \ldots$ is a valid $\left(\mathrm{OR}^{r w}\right)$-reduction, thus leaving $f($ loop $)$ semantically undefined, as would correspond to a strict semantics.

At this point, the rule (B) of CRWL is a help, since it allows to discard the evaluation of any (sub)-expression by reducing it to $\perp$. The result of this discussion is the one-step reduction relation $\longmapsto$ given in Figure 4

This relation satisfies our initial goals to a partial extent, as it is not difficult to prove the following equivalence result.

Theorem 3

Let $\mathcal{P}$ be a CRWL-program, $e \in \operatorname{Exp}_{\perp}$ and $t \in C T e r m_{\perp}$. Then:

$$
\mathcal{P} \vdash{ }_{C R W L} e \rightarrow t \text { iff } e \longmapsto_{\mathcal{P}}^{*} t
$$


This result has an interesting reading: non-strict call-time choice can be achieved via innermost evaluation if at any step one has the possibility of reducing a subexpression to $\perp$ (then, we could speak also of call-by-partial value). For instance, a $\longmapsto$-rewrite sequence with the example of Figure 1 would be:

$$
\begin{aligned}
& \text { heads }(\text { repeat }(\text { coin })) \longmapsto \text { heads }(\text { repeat }(0)) \longmapsto \\
& \text { heads }(0: \text { repeat }(0)) \longmapsto \text { heads }(0: 0 \text { : repeat }(0)) \longmapsto \\
& \text { heads }(0: 0: \perp) \longmapsto(0,0)
\end{aligned}
$$

This gives useful intuitions about non-strict call-time choice and can actually serve for a very easy implementation of it, but has a major drawback: in general, reduction of a subexpression $e$ requires a don't know guessing between $\left(\mathrm{B}^{r w}\right)$ and $\left(\mathrm{OR}^{r w}\right)$, because at the moment of reducing $e$ it is not known whether its value will be needed or not later on in the computation. Instead of reducing to $\perp$, letrewriting will create a let-binding let $U=e$ in ..., which does not imply any guessing and keeps $e$ for its eventual future use.

\section{Rewriting with local bindings}

Inspired by (Ariola and Arvind 1995, Ariola et al. 1995, Ariola and Felleisen 1997 Maraist et al. 1998: Plump 1998; Sánchez-Hernández 2004), let-rewriting extends the syntax of expressions by adding local bindings to express sharing and call-time

choice. Formally the syntax for let-expressions is:

$$
L \operatorname{Exp} \ni e::=X\left|h\left(e_{1}, \ldots, e_{n}\right)\right| \text { let } X=e_{1} \text { in } e_{2}
$$

where $X \in \mathcal{V}, h \in \Sigma^{n}$, and $e_{1}, e_{2}, \ldots, e_{n} \in L$ Exp. The intended behaviour of let $X=e_{1}$ in $e_{2}$ is that the expression $e_{1}$ will be reduced only once (at most) and then its corresponding value will be shared within $e_{2}$. For let $X=e_{1}$ in $e_{2}$ we call $e_{1}$ the definiens of $X$, and $e_{2}$ the body of the let-expression.

The sets $F V(e)$ of free and $B V(e)$ bound variables of $e \in L E x p$ are defined as:

$$
\begin{aligned}
& F V(X)=\{X\} \\
& F V(h(\bar{e}))=\bigcup_{e_{i} \in \bar{e}} F V\left(e_{i}\right) \\
& F V\left(\text { let } X=e_{1} \text { in } e_{2}\right)=F V\left(e_{1}\right) \cup\left(F V\left(e_{2}\right) \backslash\{X\}\right) \\
& B V(X)=\emptyset \\
& B V(h(\bar{e}))=\bigcup_{e_{i} \in \bar{e}} B V\left(e_{i}\right) \\
& B V\left(\text { let } X=e_{1} \text { in } e_{2}\right)=B V\left(e_{1}\right) \cup B V\left(e_{2}\right) \cup\{X\}
\end{aligned}
$$

Notice that with the given definition of $F V\left(\right.$ let $X=e_{1}$ in $\left.e_{2}\right)$ there are not recursive let-bindings in the language since the possible occurrences of $X$ in $e_{1}$ are not considered bound and therefore refer to a 'different' $X$. For example, the expression let $X=f(X)$ in $g(X)$ can be equivalently written as let $Y=f(X)$ in $g(Y)$. This is similar to what is done in (Maraist et al. 1998; Ariola et al. 1995, Ariola and Felleisen 1997), but not in (Albert et al. 2005 Launchbury 1993). Recursive lets have their own interest but there is not a general consensus in the functional logic community about their meaning in presence of non-determinism. We remark 
also that the let-bindings introduced by let-rewriting derivations to be presented in Section 4.1 are not recursive. Therefore, recursive lets are not considered in this work.

We will use the notation let $\overline{X=a}$ in $e$ as a shortcut for let $X_{1}=a_{1}$ in .. in let $X_{n}=a_{n}$ in $e$. The notion of one-hole context is also extended to the new syntax:

$$
\mathcal{C}::=[] \mid \text { let } X=\mathcal{C} \text { in } e \mid \text { let } X=e \text { in } \mathcal{C} \mid h(\ldots, \mathcal{C}, \ldots)
$$

By default, we will use contexts with lets from now on.

Free variables of contexts are defined as for expressions, so that $F V(\mathcal{C})=F V(\mathcal{C}[h])$, for any $h \in \Sigma^{0}$. However, the set $B V(\mathcal{C})$ of variables bound by a context is defined quite differently because it consists only of those let-bound variables visible from the hole of $\mathcal{C}$. Formally:

$$
\begin{aligned}
& B V([])=\emptyset \\
& B V(h(\ldots, \mathcal{C}, \ldots))=B V(\mathcal{C}) \\
& B V(\text { let } X=e \text { in } \mathcal{C})=\{X\} \cup B V(\mathcal{C}) \\
& B V(\text { let } X=\mathcal{C} \text { in } e)=B V(\mathcal{C})
\end{aligned}
$$

As a noticeable difference with respect to (López-Fraguas et al. 2007b), from now on we will allow to use lets in any program, so our program rules have the shape $f\left(p_{1}, \ldots, p_{n}\right) \rightarrow r$, for $f \in F S^{n},\left(p_{1}, \ldots, p_{n}\right)$ a linear tuple of c-terms, and $r \in L$ Exp. Notice, however, that the notion of c-term does not change: c-terms do not contain function symbols nor lets, although they can contain bound variables when put in an appropriate context as happens for example with the subexpression $(X, X)$ in the expression let $X=\operatorname{coin}$ in $(X, X)$.

As usual with syntactical binding constructs, we assume a variable convention according to which bound variables can be consistently renamed as to ensure that the same variable symbol does not occur free and bound within an expression. Moreover, to keep simple the management of substitutions, we assume that whenever $\theta$ is applied to an expression $e \in L E x p$, the necessary renamings are done in $e$ to ensure $B V(e) \cap(\operatorname{dom}(\theta) \cup \operatorname{vran}(\theta))=\emptyset$. With all these conditions the rules defining application of substitutions are simple while avoiding variable capture:

$$
\begin{aligned}
& X \theta=\theta(X), \text { for } X \in \mathcal{V} \\
& h\left(e_{1}, \ldots, e_{n}\right) \theta=h\left(e_{1} \theta, \ldots, e_{n} \theta\right), \text { for } h \in \Sigma^{n} \\
& \left(\text { let } X=e_{1} \text { in } e_{2}\right) \theta=\text { let } X=e_{1} \theta \text { in } e_{2} \theta
\end{aligned}
$$

The following example illustrates the use of these conventions.

$$
\begin{aligned}
& \text { let } X=c(X) \text { in let } Y=z \text { in } d(X, Y))[X / c(Y)] \\
& =(\text { let } U=c(X) \text { in let } V=z \text { in } d(U, V))[X / c(Y)] \\
& =\text { let } U=c(c(Y)) \text { in let } V=z \text { in } d(U, V)
\end{aligned}
$$

The following substitution lemma will be often a useful technical tool:

Lemma 1 (Substitution lemma for let-expressions)

Let $e, e^{\prime} \in L E x p_{\perp}, \theta \in S u b s t_{\perp}$ and $X \in \mathcal{V}$ such that $X \notin \operatorname{dom}(\theta) \cup \operatorname{vran}(\theta)$. Then:

$$
\left(e\left[X / e^{\prime}\right]\right) \theta \equiv e \theta\left[X / e^{\prime} \theta\right]
$$




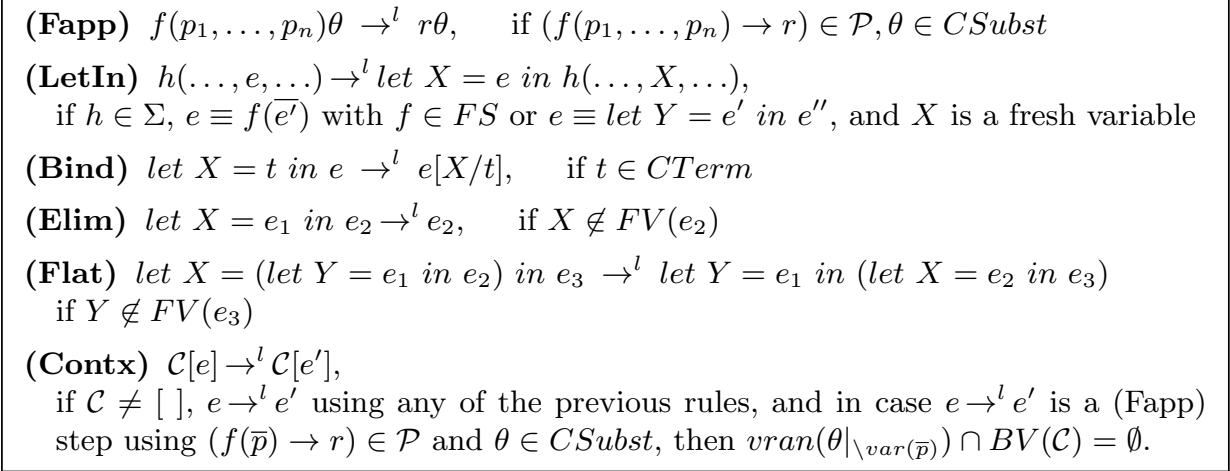

Fig. 5. Rules of the let-rewriting relation $\rightarrow^{l}$

\subsection{The let-rewriting relation}

Let-expressions can be reduced step by step by means of the let-rewriting relation $\rightarrow^{l}$, shown in Figure 5 Rule (Contx) allows us to use any subexpression as redex in the derivation. (Fapp) performs a rewriting step in the proper sense, using a program rule. Note that only c-substitutions are allowed, to avoid copying of unevaluated expressions which would destroy sharing and call-time choice. To prevent that the restriction of (Fapp) to total c-substitutions results in a strict semantics, we also provide the rule (LetIn) that suspends the evaluation of a subexpression by introducing a let-binding. If its value is needed later on, its evaluation can be performed by some (Contx) steps and the result propagated by (Bind). This latter rule is safe wrt. call-time choice because only propagates c-terms, that is, either completely defined values (without any bound variable) or partially computed values with some suspension (bound variable) on it, which will be safely managed by the calculus. On the other hand, if the bound variable disappears from the body of the let-binding during evaluation, rule (Elim) can be used for garbage collection. This rule is useful to ensure that normal forms corresponding to values are c-terms. Finally, (Flat) is needed for flattening nested lets; otherwise some reductions could become wrongly blocked or forced to diverge. Consider for example the program $\{l$ loop $\rightarrow$ loop, $g(s(X)) \rightarrow 1\}$ and the expression $g(s(l o o p))$, which can be reduced to let $X=($ let $Y=$ loop in $s(Y))$ in $g(X)$ by applying (LetIn) twice. Then, without (Flat) we could only perform reduction steps on loop, thus diverging; by using (Flat), we can obtain let $Y=$ loop in let $X=s(Y)$ in $g(X)$, which can be finally reduced to 1 by applying (Bind), (Fapp) and (Elim). The condition $Y \notin F V\left(e_{3}\right)$ in (Flat) could be dropped by the variable convention, but we have included it to keep the rules independent of the convention. Quite different is the case of (Elim), where the condition $X \notin F V\left(e_{2}\right)$ is indeed necessary.

Note that, in contrast to CRWL or the relation $\longmapsto$ in Section 3 , let-rewriting does not need to use the semantic value $\perp$, which does not appear in programs nor in computations.

Example 2

Consider the program of Figure 1 . We can perform the following let-rewriting deriva- 
tion for the expression heads(repeat(coin)), where in each step the corresponding redex has been underlined for the sake of readability.

$$
\begin{aligned}
& \text { heads(repeat }(\text { coin })) \quad \text { (LetIn) } \\
& \rightarrow{ }^{l} \text { let } X=\text { repeat }(\text { coin }) \text { in heads }(X)
\end{aligned}
$$

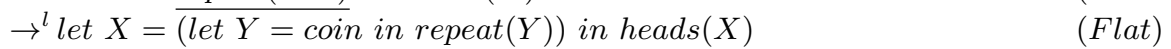

$$
\begin{aligned}
& \rightarrow l \overline{l e t} Y=\text { coin in let } X=\text { repeat }(Y) \text { in heads }(X) \quad \text { (Fapp) } \\
& \rightarrow^{l} \text { let } Y=\text { coin in let } X=\overline{Y: \text { repeat }}(Y) \text { in heads }(X) \quad \text { (LetIn) }
\end{aligned}
$$

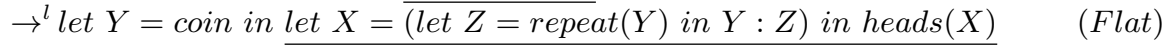

$$
\begin{aligned}
& \rightarrow^{l} \text { let } Y=\text { coin in let } Z=\text { repeat }(Y) \text { in let } X=Y: Z \text { in heads }(X) \quad \text { (Bind) } \\
& \rightarrow^{l} \text { let } Y=\text { coin in let } Z=\text { repeat }(Y) \text { in } \overline{\text { heads }(Y: Z)} \quad \text { (Fapp) } \\
& \rightarrow{ }^{l} \text { let } Y=\text { coin in let } Z=\overline{Y: \text { repeat }}(Y) \text { in heads }(Y: Z) \quad \text { (LetIn) } \\
& \left.\rightarrow^{l} \text { let } Y=\text { coin in let } Z=\overline{(\text { let } U=\operatorname{repe} a t}(Y) \text { in } Y: U\right) \text { in heads }(Y: Z) \quad \text { (Flat) } \\
& \rightarrow{ }^{l} \text { let } Y=\text { coin in let } U=\operatorname{repeat}(Y) \text { in let } Z=Y: U \text { in heads }(Y: Z) \quad \text { (Bind) } \\
& \rightarrow^{l} \text { let } Y=\text { coin in let } U=\operatorname{repeat}(Y) \text { in } \overline{\text { heads }(Y: Y: U)} \quad \text { (Fapp) } \\
& \rightarrow^{l} \text { let } Y=\text { coin in let } U=\operatorname{repeat}(Y) \text { in } \overline{(Y, Y)} \quad \text { (Elim) } \\
& \rightarrow \text { let } Y=\text { coin in } \overline{(Y, Y)} \quad \text { (Fapp) } \\
& \rightarrow{ }^{l} \text { let } Y=\overline{0 \text { in }}(Y, Y) \\
& \rightarrow^{l} \overline{(0,0)}
\end{aligned}
$$

Note that there is not a unique $\rightarrow^{l}$-reduction leading to $(0,0)$. The definition of $\rightarrow^{l}$, like traditional term rewriting, does not prescribe any particular strategy. The definition of on-demand evaluation strategies for let-rewriting is out of the scope of this paper, and is only informally discussed in Section 6.2

We study now some properties of let-rewriting that have intrinsic interest and will be useful when establishing a relation to CRWL in next sections.

The same example used in Proposition 1 to show that CRWL is not closed under general substitutions shows also that the same applies to let-rewriting. However, let-rewriting is closed under c-substitutions, as expected in a semantics for call-time choice.

Lemma 2 (Closedness under CSubst of let-rewriting) For any $e, e^{\prime} \in L \operatorname{Exp}, \theta \in C$ Subst we have that $e \rightarrow^{l^{n}} e^{\prime}$ implies $e \theta \rightarrow^{l^{n}} e^{\prime} \theta$.

Another interesting matter is the question of what happens in let-rewriting derivations in which the rule (Fapp) is not used - and as a consequence, the program is ignored.

Definition $3\left(\right.$ The $\rightarrow^{\text {lnf }}$ relation $)$

The relation $\rightarrow^{\operatorname{lnf}}$ is defined by the rules of Figure 5 except (Fapp). As a consequence, for any program $\rightarrow^{l n f} \subseteq \rightarrow^{l}$.

We can think about any let-expression $e$ as an expression from Exp in which some additional sharing information has been encoded using the let-construction. When we avoid the use of the rule (Fapp) in derivations, we do not make progress in the evaluation of the implicit let-less expression corresponding to $e$, but we change the sharing-enriched representation of that expression in the let-rewriting syntax. Following terminology from term graph rewriting — as in fact a let-expression is a textual representation of a term graph - all the rules of let-rewriting except (Fapp) 
move between two isomorphic term graphs (Plump 2001: Plump 1998). The $\rightarrow^{\operatorname{lnf}}$ relation will be used to reason about these kind of derivations.

The first interesting property of $\rightarrow^{\operatorname{lnf}}$ is that it is a terminating relation.

Proposition 2 (Termination of $\rightarrow^{\operatorname{lnf}}$ )

For any program $\mathcal{P}$, the relation $\rightarrow^{\text {lnf }}$ is terminating. As a consequence, every $e \in L E x p$ has at least one $\rightarrow^{\text {lnf }}$-normal form $e^{\prime}$ (written as $e \downarrow^{\operatorname{lnf}} e^{\prime}$ ).

However, for nontrivial signatures the relation $\rightarrow^{\operatorname{lnf}}$ is not confluent (hence the relation $\rightarrow^{l}$ is not confluent either).

Example 3

Consider a signature such that $f, g \in F S^{0}, c \in C S^{2}$ and $f \not \equiv g$. Then $c(f, g) \rightarrow^{\operatorname{lnf} f^{*}}$ let $X=f$ in let $Y=g$ in $c(X, Y)$ and $c(f, g) \rightarrow^{\operatorname{lnf} f^{*}}$ let $Y=g$ in let $X=$ $f$ in $c(X, Y)$, but these expressions do not have a common reduct.

The lack of confluence of let-rewriting is alleviated by a strong semantic property of $\rightarrow^{l n f}$ which, combined with the adequacy to CRWL of let-rewriting that we will see below, may be used as a substitute for confluence in some situations. These questions will be treated in detail in Section 4.3.1

The next result characterizes $\rightarrow^{\operatorname{lnf}}$-normal forms. What we do in $\rightarrow^{\operatorname{lnf}}$ derivations is exposing the computed part of $e$-its outer constructor part-concentrating it in the body of the resulting let, that is, the part which is not a function application whose evaluation is pending. This is why we call it 'Peeling lemma'.

Lemma 3 (Peeling lemma)

For any $e, e^{\prime} \in L \operatorname{Exp}$, if $e \downarrow^{\operatorname{lnf}} e^{\prime}$ then $e^{\prime}$ has the shape $e^{\prime} \equiv$ let $\overline{X=f(\bar{t})}$ in $e^{\prime \prime}$ such that $e^{\prime \prime} \in \mathcal{V}$ or $e^{\prime \prime} \equiv h\left(\overline{t^{\prime}}\right)$ with $h \in \Sigma, \bar{f} \subseteq F S$ and $\bar{t}, \overline{t^{\prime}} \subseteq C T e r m$.

Moreover if $e \equiv h\left(e_{1}, \ldots, e_{n}\right)$ with $h \in \Sigma$, then

$$
e \equiv h\left(e_{1}, \ldots, e_{n}\right) \rightarrow^{\operatorname{lnf} f^{*}} \text { let } \overline{X=f(\bar{t})} \text { in } h\left(t_{1}, \ldots, t_{n}\right) \equiv e^{\prime}
$$

under the conditions above, and verifying also that $t_{i} \equiv e_{i}$ whenever $e_{i} \in C T e r m$.

The next property of $\rightarrow^{l}$ and $\rightarrow^{l n f}$ uses the notion of shell $|e|$ of an expression $e$, that is the partial c-term corresponding to the outer constructor part of $e$. More precisely:

Definition 4 (Shell of a let-expression)

$$
\begin{aligned}
& |X| \quad=X \quad \text { for } X \in \mathcal{V} \\
& \left|c\left(e_{1}, \ldots, e_{n}\right)\right| \quad=c\left(\left|e_{1}\right|, \ldots,\left|e_{n}\right|\right) \quad \text { for } c \in C S \\
& \left|f\left(e_{1}, \ldots, e_{n}\right)\right| \quad=\perp \quad \text { for } f \in F S \\
& \mid \text { let } X=e_{1} \text { in } e_{2}|=| e_{2} \mid\left[X /\left|e_{1}\right|\right]
\end{aligned}
$$

Notice that in the case of a let-rooted expression, the information contained in the binding is taken into account for building up the shell of the whole expression: for instance $\mid c($ let $X=2$ in $s(X)) \mid=c(s(2))$.

During a computation, the evolution of shells reflects the progress towards a value. The next result shows that shells never decrease. Moreover, only (Fapp) may 
change shells. As discussed above, 'peeling' steps (i.e. $\rightarrow^{\operatorname{lnf}}$ - steps) just modify the representation of the implicit term graph corresponding to a let-expression; thus, they preserve the shell.

\section{Lemma 4 (Growing of shells)}

i) $e \rightarrow^{l^{*}} e^{\prime}$ implies $|e| \sqsubseteq\left|e^{\prime}\right|$, for any $e, e^{\prime} \in L \operatorname{Exp}$

ii) $e \rightarrow^{l n f^{*}} e^{\prime}$ implies $|e| \equiv\left|e^{\prime}\right|$, for any $e, e^{\prime} \in L E x p$

\subsection{The $C R W L_{\text {let }}$ logic}

In this section we extend the CRWL logic to deal with let-expressions, obtaining an enlarged framework that will be useful as a bridge to establish the connection between CRWL and let-rewriting.

As in the CRWL framework, we consider partial let-expressions $e \in L E x p_{\perp}$, defined in the natural way. The approximation order $\sqsubseteq$ is also extended to $L E x p_{\perp}$ but now using the notion of context for let-expressions, which in particular implies that let $X=e_{1}$ in $e_{2} \sqsubseteq$ let $X=e_{1}^{\prime}$ in $e_{2}^{\prime}$ iff $e_{1} \sqsubseteq e_{1}^{\prime}$ and $e_{2} \sqsubseteq e_{2}^{\prime}$. The CRWL Tet $_{\text {let }}$ logic results of adding the following rule (Let) to the CRWL logic of Section 2.2.

$$
\text { (Let) } \frac{e_{1} \rightarrow t_{1} \quad e_{2}\left[X / t_{1}\right] \rightarrow t}{\text { let } X=e_{1} \text { in } e_{2} \rightarrow t}
$$

We write $\mathcal{P} \vdash_{C R W L_{l e t}} e \rightarrow t$ if $e \rightarrow t$ is derivable in the $\mathrm{CRWL}_{\text {let }}$-calculus using the program $\mathcal{P}$. In many occasions, we will omit $\mathcal{P}$.

Definition 5 (CRWL $_{\text {let-denotation) }}$

Given a program $\mathcal{P}$, the $C R W L_{\text {let }}$-denotation of $e \in L E x p_{\perp}$ is defined as:

$$
\llbracket e \rrbracket_{C R W L_{\text {let }}}^{\mathcal{P}}=\left\{t \in C T e r m_{\perp} \mid \mathcal{P} \vdash_{C R W L_{\text {let }}} e \rightarrow t\right\}
$$

We will omit the sub(super)-scripts when they are clear by the context.

There is an obvious relation between CRWL and $\mathrm{CRWL}_{l e t}$ for programs and expressions without lets:

Theorem 4 (CRWL vs. CRWL let $)$

For any program $\mathcal{P}$ without lets, and any $e \in \operatorname{Exp}_{\perp}$ :

$$
\llbracket e \rrbracket_{C R W L}^{\mathcal{P}}=\llbracket e \rrbracket_{C R W L_{l e t}}^{\mathcal{P}}
$$

This result allows us to skip the mention to CRWL or $\mathrm{CRWL}_{l e t}$ when referring to the denotation $\llbracket e \rrbracket$ of an expression: if some let-binding occurs in $e$ - or in the program wrt. which the denotation is considered - then $\llbracket e \rrbracket$ can be interpreted only as $\llbracket e \rrbracket_{C R W L_{l e t}}$; otherwise, both denotations coincide.

The $\mathrm{CRWL}_{\text {let }}$ logic inherits from CRWL a number of useful properties.

Lemma 5

For any program $e \in L E x p_{\perp}, t, t^{\prime} \in C T e r m_{\perp}$ :

i) $t \rightarrow t^{\prime}$ iff $t^{\prime} \sqsubseteq t$.

ii) $|e| \in \llbracket e \rrbracket$. 
iii) $\llbracket e \rrbracket \subseteq(|e| \uparrow) \downarrow$, where for a given $E \subseteq L E x p_{\perp}$ its upward closure is $E \uparrow=\left\{e^{\prime} \in L E x p_{\perp} \mid \exists e \in E . e \sqsubseteq e^{\prime}\right\}$, its downward closure is $E \downarrow=$ $\left\{e^{\prime} \in L E x p_{\perp} \mid \exists e \in E . e^{\prime} \sqsubseteq e\right\}$, and those operators are overloaded for let-expressions as $e \uparrow=\{e\} \uparrow$ and $e \downarrow=\{e\} \downarrow$.

The first part of the previous result shows that c-terms can only be reduced to smaller c-terms. The other parts express that the shell of an expression represents 'stable' information contained in the expression in a similar way to Lemma 4, as the shell is in the denotation by ii), and everything in the denotation comes from refining it by $i i i)$.

The following results are adaptations to $\mathrm{CRWL}_{l e t}$ of properties known for CRWL (González-Moreno et al. 1999, Vado-Vírseda 2002). The first one states that if we can compute a value for an expression then from greater expressions we can reach smaller values. The second one says that $\mathrm{CRWL}_{l e t}$-derivability is closed for partial c-substitutions.

Proposition 3 (Polarity of $C R W L_{l e t}$ )

For any $e, e^{\prime} \in L E x p_{\perp}, t, t^{\prime} \in C T e r m_{\perp}$, if $e \sqsubseteq e^{\prime}$ and $t^{\prime} \sqsubseteq t$ then $e \rightarrow t$ implies $e^{\prime} \rightarrow t^{\prime}$ with a proof of the same size or smaller - where the size of a CRWL $\mathrm{C}_{l e t}$-proof is measured as the number of rules of the calculus used in the proof.

Proposition 4 (Closedness under c-substitutions of $C R W L_{l e t}$ )

For any $e \in L E x p_{\perp}, t \in C$ Term $_{\perp}, \theta \in C S u b s t_{\perp}, t \in \llbracket e \rrbracket$ implies $t \theta \in \llbracket e \theta \rrbracket$.

Compositionality is a more delicate issue. Theorem 1 does not hold for $\mathrm{CRWL}_{l e t}$, as shown by the following example: consider the program $\{f(0) \rightarrow 1\}$, the expression $e \equiv f(X)$ and the context $\mathcal{C} \equiv$ let $X=0$ in []$. \mathcal{C}[e]$ can produce the value 1 . However, $f(X)$ can only be reduced to $\perp$, and $\mathcal{C}[\perp]$ cannot reach the value 1 . The point in that example is that the subexpression $e$ needs some information from the context to produce a value that is then used by the context to compute the value for the whole expression $\mathcal{C}[e]$. This information may only be the definientia of some variables of $e$ that get bound when put in $\mathcal{C}$; with this idea in mind we can state the following weak compositionality result for $\mathrm{CRWL}_{l e t}$.

Theorem 5 (Weak Compositionality of $C R W L_{l e t}$ )

For any $\mathcal{C} \in$ Cntxt, $e \in L \operatorname{Exp}_{\perp}$

$$
\llbracket \mathcal{C}[e] \rrbracket=\bigcup_{t \in \llbracket e \rrbracket} \llbracket \mathcal{C}[t] \rrbracket \quad \text { if } B V(\mathcal{C}) \cap F V(e)=\emptyset
$$

As a consequence, $\llbracket$ let $X=e_{1}$ in $e_{2} \rrbracket=\bigcup_{t_{1} \in \llbracket e_{1} \rrbracket} \llbracket e_{2}\left[X / t_{1}\right] \rrbracket$.

In spite of not being a fully general compositionality result, Theorem 5 can be used to prove new properties of $\mathrm{CRWL}_{l e t}$, like the following monotonicity property related to substitutions, that will be used later on. It is formulated for the partial order $\sqsubseteq$ over $L S u b s t_{\perp}$ (defined naturally as it happened for $S u s b t_{\perp}$ ) and the preorder $\unlhd$ over $L S u b s t_{\perp}$, defined by $\sigma \unlhd \sigma^{\prime}$ iff $\forall X \in \mathcal{V}, \llbracket \sigma(X) \rrbracket \subseteq \llbracket \sigma^{\prime}(X) \rrbracket$. 
Proposition 5 (Monotonicity for substitutions of $C R W L_{l e t}$ )

If $\sigma \sqsubseteq \sigma^{\prime}$ or $\sigma \unlhd \sigma^{\prime}$ then $\llbracket e \sigma \rrbracket \subseteq \llbracket e \sigma^{\prime} \rrbracket$, for any $e \in L E x p_{\perp}$ and $\sigma, \sigma^{\prime} \in L S u b s t_{\perp}$.

The limitations of Theorem 5 make us yearn for another semantic notion for letexpressions with a better compositional behaviour. We have already seen that the problem with $\mathrm{CRWL}_{l e t}$ is the possible loss of definientia when extracting an expression from its context. But in fact what bound variables need is access to the values of their corresponding definientia, as it is done in the rule (Let) where the value of the definiens is transmited to the body of the let-binding by applying a c-substitution replacing the bound variable by that value. With these ideas in mind we define the stronger notion of hyperdenotation (sometimes we say hypersemantics), which gives a more active role to variables in expressions: in contrast to the denotation of an expression $e$, which is a set of c-terms, its hyperdenotation $\llbracket e \rrbracket$ is a function mapping c-substitutions to denotations, i.e., to sets of c-terms.

\section{Definition 6 (Hyperdenotation)}

The hyperdenotation of an expression $e \in L E x p_{\perp}$ under a program $\mathcal{P}$ is a function $\llbracket e \rrbracket^{\mathcal{P}}: C S u b s t_{\perp} \rightarrow$ Den defined by $\llbracket e \rrbracket^{\mathcal{P}} \theta=\llbracket e \theta \rrbracket^{\mathcal{P}}$.

As usual, in most cases we will omit the mention to $\mathcal{P}$. We will use sometimes $H D$ as an alias for $C S u b s t_{\perp} \rightarrow$ Den, i.e, for the kind of objects that are hyperdenotations of expressions.

The notion of hyperdenotation is strictly more powerful than the notion of $\mathrm{CRWL}_{\text {let }}$ denotation. Equality of hyperdenotations implies equality of denotations -because if $\llbracket e \rrbracket=\llbracket e^{\prime} \rrbracket$ then $\llbracket e \rrbracket=\llbracket e \epsilon \rrbracket=\llbracket e \rrbracket \epsilon=\llbracket e^{\prime} \rrbracket \epsilon=\llbracket e^{\prime} \epsilon \rrbracket=\llbracket e^{\prime} \rrbracket$ - but the opposite does not hold: consider the program $\{f(0) \rightarrow 1\}$ and the expressions $f(X)$ and $\perp$; they have the same denotation (the set $\{\perp\}$ ) but different hyperdenota-

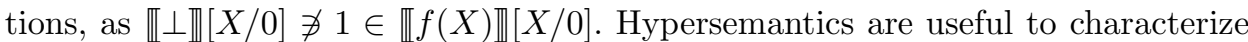
the meaning of expressions present in a context in which some of its variables may get bound, like in the body of a let-binding or in the right hand side of a program rule. Therefore are useful to reason about expressions put in arbitrary contexts, in which let-bindings may freely appear.

Most remarkably, hyperdenotations allow to recover strong compositionality results for let-expressions similar to Theorems 1 and 2. We find more intuitive to start the analogous to the latter. Semantics of contexts were defined as denotation transformers (Definition 2). Analogously, the hypersemantics $\llbracket \mathcal{C} \rrbracket$ of a context $\mathcal{C}$ is a hyperdenotation transformer defined as follows:

Definition 7 (Hypersemantics of a context)

Given $\mathcal{C} \in C$ ntxt, its hyperdenotation is a function $\llbracket \mathcal{C} \rrbracket: H D \rightarrow H D$ defined by induction over the structure of $\mathcal{C}$ as follows:

- $\llbracket[] \rrbracket \varphi \theta=\varphi \theta$

- $\llbracket h\left(e_{1}, \ldots, \mathcal{C}, \ldots, e_{n}\right) \rrbracket \varphi \theta=\bigcup_{t \in \llbracket \mathcal{C} \rrbracket \varphi \theta} \llbracket h\left(e_{1} \theta, \ldots, t, \ldots, e_{n} \theta\right) \rrbracket$

- $\llbracket$ let $X=\mathcal{C}$ in $e \rrbracket \varphi \theta=\bigcup_{t \in \llbracket \mathcal{C} \rrbracket \varphi \theta} \llbracket$ let $X=t$ in $e \theta \rrbracket$

- $\llbracket$ let $X=e$ in $\mathcal{C} \rrbracket \varphi \theta=\bigcup_{t \in \llbracket e \rrbracket \theta}^{\llbracket} \llbracket \mathcal{C} \rrbracket \varphi(\theta[X / t])$ 
With this notion, our first version of strong compositionality for hypersemantics looks like Theorem 2 .

Theorem 6 (Compositionality of hypersemantics)

For all $\mathcal{C} \in C$ ntxt, $e \in L E x p_{\perp}$

$$
\llbracket \mathcal{C}[e] \rrbracket=\llbracket \mathcal{C} \rrbracket \llbracket e \rrbracket
$$

As a consequence: $\llbracket e \rrbracket=\llbracket e^{\prime} \rrbracket \Leftrightarrow \forall \mathcal{C} \in$ Cntxt. $\llbracket \mathcal{C}[e] \rrbracket=\llbracket \mathcal{C}\left[e^{\prime}\right] \rrbracket$.

This result implies that in any context we can replace any subexpression by another one having the same hypersemantics (and therefore also the same semantics) without changing the hypersemantics (hence the semantics) of the global expression.

In Theorems 2 and 6 the role of call-time choice is hidden in the definition of semantics and hypersemantics of a context, respectively. To obtain a version of strong compositionalty of hypersemantics closer to Theorem 1 and 5 , we need some more notions and notations about hyperdenotations or, more generally, about functions in $H D$. Since they are set-valued functions, many usual set operations and relations can be lifted naturally in a pointwise manner to $H D$. The precise definitions become indeed clearer if we give them for general sets, abstracting away the details about $H D$. We introduce also some notions about decomposing set-valued functions that will be useful for hyperdenotations. We use freely $\lambda$-notation to write down a function in the mathematical sense; we may write $\lambda x \in A$ to indicate its domain $A$, if it not clear by the context.

Definition 8 (Operations and relations for set-valued functions)

Let $A, B$ be two sets, $\mathcal{F}$ the set of functions $A \rightarrow \mathcal{P}(B)$, and $f, g \in \mathcal{F}$. Then:

i) The hyperunion of $f, g$ is defined as $f \cup g=\lambda x \in A . f(x) \cup g(x)$.

ii) More generally, the hyperunion of a family $\mathcal{I} \subseteq \mathcal{F}$, written indistinctly as UU $\mathcal{I}$ or $\underset{f \in \mathcal{I}}{\bigcup} f$, is defined as

$$
\text { UU } \mathcal{I} \equiv \bigcup_{f \in \mathcal{I}} f=\operatorname{def}_{\text {def }} \quad \lambda x \in A . \bigcup_{f \in \mathcal{I}} f(x)
$$

Notice that $f \cup g=\bigcup\{\{f, g\}$.

iii) We say that $f$ is hyperincluded in $g$, written $f \Subset g$, iff $\forall x \in A . f(x) \subseteq g(x)$.

iv) A decomposition of $f$ is any $\mathcal{I} \subseteq \mathcal{F}$ such that $\bigcup \mathcal{I}=f$.

v) The elemental decomposition of $f$ is the following set of functions of $\mathcal{F}$ :

$$
\Delta f=\left\{\lambda x \in A .\left\{\begin{array}{l}
\{b\} \text { if } x=a \\
\emptyset \text { otherwise }
\end{array} \quad \mid a \in A, b \in f(a)\right\}\right.
$$

Or, using the abbreviation $\hat{\lambda} a \cdot\{b\}$ as a shorthand for $\lambda x \cdot\left\{\begin{array}{c}\{b\} \text { if } x=a \\ \emptyset \text { otherwise }\end{array}\right.$

$$
\Delta f=\{\hat{\lambda} a \cdot\{b\} \mid a \in A, b \in f(a)\}
$$


Decompositions are used to split set-valued functions into smaller pieces; elemental decompositions do it with minimal ones. For instance, if $f:\{a, b\} \rightarrow \mathcal{P}(\{0,1,2\})$ is given by $f(a)=\{0,2\}$ and $f(b)=\{1,2\}$, then $\Delta f=\{\hat{\lambda} a .\{0\}, \hat{\lambda} a .\{2\}, \hat{\lambda} b .\{1\}, \hat{\lambda} b .\{2\}\}$.

Hyperinclusion and hyperunion share many properties of standard set inclusion and union. Some of them are collected in the next result, that refer also to decompositions:

\section{Proposition 6}

Consider two sets $A, B$, and let $\mathcal{F}$ be the set of functions $A \rightarrow \mathcal{P}(B)$. Then:

i) $\Subset$ is indeed a partial order on $\mathcal{F}$, and $\Delta f$ is indeed a decomposition of $f \in \mathcal{F}$, i.e., $U$ U $(\Delta f)=f$.

ii) Monotonicity of hyperunion wrt. inclusion: for any $\mathcal{I}_{1}, \mathcal{I}_{2} \subseteq \mathcal{F}$

$$
\mathcal{I}_{1} \subseteq \mathcal{I}_{2} \text { implies } U U \mathcal{I}_{1} \Subset U \mathcal{I}_{2}
$$

iii) Distribution of unions: for any $\mathcal{I}_{1}, \mathcal{I}_{2} \subseteq \mathcal{F}$

$$
\text { UU }\left(\mathcal{I}_{1} \cup \mathcal{I}_{2}\right)=\left(\bigcup \mathcal{I}_{1}\right) \cup\left(\bigcup \mathcal{I}_{2}\right)
$$

iv) Monotonicity of decomposition wrt. hyperinclusion: for any $f_{1}, f_{2} \in \mathcal{F}$

$$
f_{1} \Subset f_{2} \text { implies } \Delta f_{1} \subseteq \Delta f_{2}
$$

We will apply all these notions, notations and properties to the case when $A \equiv$ $C S u b s t_{\perp}$ and $B \equiv C T e r m_{\perp}$ (i.e. $\mathcal{P}(B) \equiv D e n$ and therefore $\mathcal{F} \equiv H D$ ). Therefore, we can speak of the hyperunion of two hyperdenotations, or of a family of them, we can elementarily decompose a hyperdenotation, etc.

Proposition 7 (Distributivity under context of hypersemantics unions)

$$
\llbracket \mathcal{C} \rrbracket(\bigcup H)=\bigcup_{\varphi \in H} \llbracket \mathcal{C} \rrbracket \varphi
$$

With this result we can easily prove our desired new version of a strong compositionality result for hypersemantics, with a style closer to the formulations of Theorems 1 and 5 . This new form of compositionality will be used in Section 5.1 for building a straightforward proof of the adequacy of a transformation that otherwise becomes highly involved by using other techniques.

Theorem 7 (Compositionality of hypersemantics, version 2)

For any $\mathcal{C} \in C$ ntxt, $e \in \operatorname{LExp}_{\perp}$ :

$$
\llbracket \mathcal{C}[e] \rrbracket=\bigcup_{\varphi \in H} \llbracket \mathcal{C} \rrbracket \varphi, \text { for any decomposition } H \text { of } \llbracket e \rrbracket
$$

In particular: $\llbracket \mathcal{C}[e] \rrbracket=\underset{\varphi \in \Delta \llbracket e \rrbracket}{\bigcup} \llbracket \mathcal{C} \rrbracket \varphi$.

As a consequence: $\llbracket e \rrbracket=\llbracket e^{\prime} \rrbracket \Leftrightarrow \forall \mathcal{C} \in C n t x t . \llbracket \mathcal{C}[e] \rrbracket=\llbracket \mathcal{C}\left[e^{\prime}\right] \rrbracket$. 
Proof

$$
\begin{array}{ll}
\llbracket \mathcal{C}[e] \rrbracket=\llbracket \mathcal{C} \rrbracket \llbracket e \rrbracket & \text { by compositionality v.1 (Theorem 6] } \\
=\llbracket \mathcal{C} \rrbracket(\bigcup U H) & \text { by definition of decomposition (Def. 8 } i v \text { ) } \\
=\bigcup_{\varphi \in H} \llbracket \mathcal{C} \rrbracket \varphi & \text { by distributivity (Proposition } 7)
\end{array}
$$

As happened with Theorems 1 and 2 with respect to denotations, Theorems 6 and 7 are different aspects of the same property, which shows that the hypersemantics of a whole let-expression depends only on the hypersemantics of its constituents; it also allows us to interchange in a context any pair of expressions with the same hypersemantics. This is reflected on the fact that we have attached $\llbracket e \rrbracket=\llbracket e^{\prime} \rrbracket \Leftrightarrow \forall \mathcal{C} \in C n t x t . \llbracket \mathcal{C}[e] \rrbracket=\llbracket \mathcal{C}\left[e^{\prime}\right] \rrbracket$ as a trivial consequence both in Theorem 6 and Theorem 7. Moreover, Theorem 6 can also be proved by a combination of Theorem 7 and Propositions 6 i) and 7 , in a similar way to the proof for Theorem 7] above.

$$
\begin{array}{ll}
\llbracket \mathcal{C}[e] \rrbracket=\underset{\varphi \in H}{U} \llbracket \mathcal{C} \rrbracket \varphi & \text { by compositionality v.2 (Theorem } 7) \\
=\llbracket \mathcal{C} \rrbracket(\bigcup U(\Delta \llbracket e \rrbracket)) & \text { by distributivity (Proposition } 7) \\
=\llbracket \mathcal{C} \rrbracket \llbracket e \rrbracket & \text { because } \Delta \llbracket e \rrbracket \text { decomposes } e \text { (Proposition 6 } i) \text { ) }
\end{array}
$$

Therefore Theorems 6 and 7 are results with the same strength, two sides of the same coin that will be useful tools for reasoning with hypersemantics.

To conclude, we present the following monotonicity property under contexts of hypersemantics, which will be useful in the next section.

Lemma 6 (Monotonicity under contexts of hypersemantics)

For any $\mathcal{C} \in$ Cntxt, $\varphi_{1}, \varphi_{2} \in H D$ :

$$
\varphi_{1} \Subset \varphi_{2} \text { implies that } \llbracket \mathcal{C} \rrbracket \varphi_{1} \Subset \llbracket \mathcal{C} \rrbracket \varphi_{2}
$$

Proof

Assume $\varphi_{1} \Subset \varphi_{2}$. Then:

$$
\begin{aligned}
& \left.\llbracket \mathcal{C} \rrbracket \varphi_{1}=\llbracket \mathcal{C} \rrbracket\left(\bigcup \int\left(\Delta \varphi_{1}\right)\right) \quad \text { by Proposition 6 } i\right)
\end{aligned}
$$

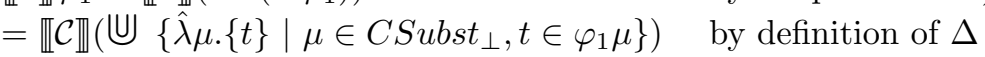

$$
\begin{aligned}
& \Subset \llbracket \mathcal{C} \rrbracket\left(\bigcup\left\{\hat{\lambda} \mu .\{t\} \mid \mu \in C S u b s t_{\perp}, t \in \varphi_{2} \mu\right\}\right) \quad \text { by Proposition 6 ii) } \\
& =\llbracket \mathcal{C} \rrbracket\left(U\left(\Delta \varphi_{2}\right)\right) \quad \text { by definition of } \Delta \\
& =\llbracket \mathcal{C} \rrbracket \varphi_{2} \quad \text { by Proposition } 6 \text { i) }
\end{aligned}
$$

We have now the tools needed to tackle the task of formally relating CRWL and let-rewriting.

\subsection{Equivalence of let-rewriting to $C R W L$ and $C R W L_{l e t}$}

In this section we prove soundness and completeness results of let-rewriting with respect to $\mathrm{CRWL}_{l e t}$ and CRWL. 


\subsubsection{Soundness}

Concerning soundness we want to prove that $\rightarrow^{l}$-steps do not create new CRWLsemantic values. More precisely:

Theorem 8 (Soundness of let-rewriting)

For all $e, e^{\prime} \in L$ Exp, if $e \rightarrow^{l^{*}} e^{\prime}$ then $\llbracket e^{\prime} \rrbracket \subseteq \llbracket e \rrbracket$.

Notice that because of non-determinism $\subseteq$ cannot be replaced by $=$ in this theorem. For example, with the program $\mathcal{P}=\{$ coin $\rightarrow 0$, coin $\rightarrow 1\}$ we can perform the step $\operatorname{coin} \rightarrow^{l} 0$, for which $\llbracket 0 \rrbracket=\{0, \perp\}, \llbracket \operatorname{coin} \rrbracket=\{0,1, \perp\}$.

It is interesting to explain why a direct reasoning with denotations fails to prove Theorem 8

A proof could proceed straightforwardly by a case distinction on the rules for $\rightarrow^{l}$ to prove the soundness of a single $\rightarrow^{l}$ step. The problem is that the case for a (Contx) step would need the following monotonicity property under context of $\mathrm{CRWL}_{\text {let }}$ denotations:

$$
\llbracket e \rrbracket \subseteq \llbracket e^{\prime} \rrbracket \text { implies } \llbracket \mathcal{C}[e] \rrbracket \subseteq \llbracket \mathcal{C}\left[e^{\prime}\right] \rrbracket
$$

Unfortunately, the property is false, for the same reasons that already explained the weakness of Theorem 5 , the possible capture of variables when switching from $e$ to $\mathcal{C}[e]$.

\section{Counterexample 1}

Consider the program $\mathcal{P}=\{f(0) \rightarrow 1\}$. We have $\llbracket f(X) \rrbracket=\{\perp\} \subseteq\{\perp, 0\}=\llbracket 0 \rrbracket$, but when these expressions are placed within the context let $X=0$ in [ ] we obtain $\llbracket$ let $X=0$ in $f(X) \rrbracket=\{\perp, 1\} \nsubseteq\{\perp, 0\}=\llbracket$ let $X=0$ in $0 \rrbracket$.

The good thing is that we can overcome these problems by using hypersemantics. Theorem 8 will be indeed an easy corollary of the following generalization to hypersemantics.

Theorem 9 (Hyper-Soundness of let-rewriting) For all $e, e^{\prime} \in L$ Exp, if $e \rightarrow^{l^{*}} e^{\prime}$ then $\llbracket e^{\prime} \rrbracket \Subset \llbracket e \rrbracket$.

And, in order to prove this generalized theorem, we also devise a generalization of the faulty monotonicity property of $\mathrm{CRWL}_{l e t}$ denotations above mentioned. That generalization is an easy consequence of the compositionality and monotonicity under contexts of hypersemantics.

Lemma 7

For all $e, e^{\prime} \in L \operatorname{Exp}_{\perp}$ and $\mathcal{C} \in C n t x t$, if $\llbracket e \rrbracket \Subset \llbracket e^{\prime} \rrbracket$ then $\llbracket \mathcal{C}[e] \rrbracket \Subset \llbracket \mathcal{C}\left[e^{\prime}\right] \rrbracket$.

Proof

$\begin{array}{ll}\llbracket \mathcal{C}[e] \rrbracket=\llbracket \mathcal{C} \rrbracket \llbracket e \rrbracket & \text { by Theorem } 6 \\ \Subset \llbracket \mathcal{C} \rrbracket \llbracket e^{\prime} \mathbb{} & \text { by Lemma } 6, \text { as } \llbracket e \rrbracket \Subset \llbracket e^{\prime} \rrbracket \\ =\llbracket \mathcal{C}\left[e^{\prime}\right] \rrbracket & \text { by Theorem } 6\end{array}$


With the help of Lemma 7, we can now prove Theorem 9 by a simple case distinction on the rules for $\rightarrow^{l}$ and a trivial induction on the length of the derivation. Now, Theorem 8 follows as an easy consequence.

Proof for Theorem 8

Assume $e \rightarrow^{l^{*}} e^{\prime}$. By Theorem 9 we have $\llbracket e^{\prime} \rrbracket \Subset \llbracket e \rrbracket$, and therefore $\llbracket e^{\prime} \theta \rrbracket \subseteq \llbracket e \theta \rrbracket$ for every $\theta \in C S u b s t_{\perp}$. Choosing $\theta=\epsilon$ (the empty substitution) we obtain $\llbracket e^{\prime} \rrbracket \subseteq \llbracket e \rrbracket$ as desired.

The moral then is that when reasoning about the semantics of expressions and programs with lets it is usually better to lift the problem to the hypersemantic world, and then particularize to semantics the obtained result. This is done, for instance, in the following result:

Proposition 8 (The $\rightarrow{ }^{\operatorname{lnf}}$ relation preserves hyperdenotation)

For all $e, e^{\prime} \in L E x p$, if $e \rightarrow^{l n f^{*}} e^{\prime}$ then $\llbracket e \rrbracket=\llbracket e^{\prime} \rrbracket$ - and therefore $\llbracket e \rrbracket=\llbracket e^{\prime} \rrbracket$.

This result mirrors semantically the fact that $\rightarrow^{\operatorname{lnf}}$ performs transitions between let-expressions corresponding to the same implicit term graph. Proposition 8 in some sense lessens the importance of the lack of confluence for the $\rightarrow{ }^{\ln f}$ relation seen in Section 4.1. Preservation of hyperdenotation may be used in some situations as a substitute for confluence, specially taking into account that let-rewriting and $\mathrm{CRWL}_{l e t}$ enjoy a really strong equivalence, as it is shown in this section.

Finally, we combine the previous results in order to get our main result concerning the soundness of let-rewriting with respect to the $\mathrm{CRWL}_{l e t}$ calculus:

Theorem 10 (Soundness of let-rewriting)

For any program $\mathcal{P}$ and $e \in L$ Exp we have:

i) $e \rightarrow^{l *} e^{\prime}$ implies $\mathcal{P} \vdash_{C R W L_{l e t}} e \rightarrow\left|e^{\prime}\right|$, for any $e^{\prime} \in L E x p$.

ii) $e \rightarrow^{l *} t$ implies $\mathcal{P} \vdash_{C R W L_{\text {let }}} e \rightarrow t$, for any $t \in C T e r m$.

Furthermore, if neither $\mathcal{P}$ nor $e$ have lets then we also have:

iii) $e \rightarrow^{l *} e^{\prime}$ implies $\mathcal{P} \vdash_{C R W L} e \rightarrow\left|e^{\prime}\right|$, for any $e^{\prime} \in L E x p$.

iv) $e \rightarrow^{l^{*}} t$ implies $\mathcal{P} \vdash_{C R W L} e \rightarrow t$, for any $t \in C T e r m$.

\section{Proof}

i) Assume $e \rightarrow^{l *} e^{\prime}$. Then, by Theorem 8 we have $\llbracket e^{\prime} \rrbracket_{C R W L_{l e t}} \subseteq \llbracket e \rrbracket_{C R W L_{l e t}}$. Since $\left|e^{\prime}\right| \in \llbracket e^{\prime} \rrbracket_{C R W L_{l e t}}$ by Lemma 5, we get $\left|e^{\prime}\right| \in \llbracket e \rrbracket_{C R W L_{l e t}}$, which means $e \rightarrow\left|e^{\prime}\right|$.

ii) Trivial by $(i)$, since $|t|=t$ for any $t \in C$ Term.

iii) Just combining $i$ ) and Theorem 4 .

iv) Just combining ii) and Theorem 4 . 


\subsubsection{Completeness}

Now we look for the reverse implication of Theorem 10, that is, the completeness of let-rewriting as its ability to compute, for any given expression, any value that can been computed by the CRWL-calculi. With the aid of the Peeling Lemma 3 we can prove the following strong completeness result for let-rewriting, which still has a certain technical nature.

Lemma 8 (Completeness lemma for let-rewriting)

For any $e \in L E x p$ and $t \in C$ Term $_{\perp}$ such that $t \not \equiv \perp$,

$$
e \rightarrow t \text { implies } e \rightarrow^{l^{*}} \text { let } \overline{X=a} \text { in } t^{\prime}
$$

for some $t^{\prime} \in C$ Term and $\bar{a} \subseteq L$ Exp such that $t \sqsubseteq \mid$ let $\overline{X=a}$ in $t^{\prime} \mid$ and $\left|a_{i}\right|=\perp$ for every $a_{i} \in \bar{a}$. As a consequence, $\left.t \sqsubseteq t^{\prime} \overline{X / \perp}\right]$.

Note the condition $t \not \equiv \perp$ is essential for this lemma to be true, as we can see by taking $\mathcal{P}=\{$ loop $\rightarrow$ loop $\}$ and $e \equiv$ loop: while loop $\rightarrow \perp$, the only $L E x p$ reachable from loop is loop itself.

Our main result concerning completeness of let-rewriting follows easily from Lemma 8. It shows that any c-term computed by CRWL or CRWL $\mathrm{Clet}_{\text {for }}$ an expression can be refined by a let-rewriting derivation; moreover, if the c-term is total, then it can be exactly reached by let-rewriting.

Theorem 11 (Completeness of let-rewriting)

For any program $\mathcal{P}, e \in L E x p$, and $t \in C T e r m_{\perp}$ we have:

i) $\mathcal{P} \vdash_{C R W L_{\text {let }}} e \rightarrow t$ implies $e \rightarrow^{l *} e^{\prime}$ for some $e^{\prime} \in L E x p$ such that $t \sqsubseteq\left|e^{\prime}\right|$

ii) Besides, if $t \in C$ Term then $\mathcal{P} \vdash_{C R W L_{l e t}} e \rightarrow t$ implies $e \rightarrow^{l *} t$

Furthermore, if neither $\mathcal{P}$ nor $e$ have lets then we also have

iii) $\mathcal{P} \vdash_{C R W L} e \rightarrow t$ implies $e \rightarrow^{l *} e^{\prime}$ for some $e^{\prime} \in L$ Exp such that $t \sqsubseteq\left|e^{\prime}\right|$

iv) Besides, if $t \in C$ Term then $\mathcal{P} \vdash_{C R W L} e \rightarrow t$ implies $e \rightarrow^{l^{*}} t$

Proof

Regarding part $i$ ), if $t \equiv \perp$ then we are done with $e \rightarrow^{l}{ }^{0} e$ as $\forall e, \perp \sqsubseteq|e|$. On the other hand, if $t \not \equiv \perp$ then by Lemma 8 we have $e \rightarrow^{l *}$ let $\bar{X}=\bar{a}$ in $t^{\prime}$ such that $t \sqsubseteq \mid$ let $\bar{X}=\bar{a}$ in $t^{\prime} \mid$

To prove part ii), assume $\mathcal{P} \vdash_{C R W L_{l e t}} e \rightarrow t$. Then, by Lemma 8 , we get $e \rightarrow{ }^{*}$ let $\bar{X}=\bar{a}$ in $t^{\prime}$ such that $t \sqsubseteq \mid$ let $\bar{X}=\bar{a}$ in $t \mid \equiv t^{\prime}[\bar{X} / \bar{\perp}]$, for some $t^{\prime} \in$ $C T e r m, \bar{a} \subseteq L$ Exp. As $t \in C$ Term then $t$ is maximal wrt. $\sqsubseteq$, so $t \sqsubseteq t^{\prime}[\bar{X} / \bar{\perp}]$ implies $t^{\prime}[\bar{X} / \bar{\perp}] \equiv t$, but then $t^{\prime}[\bar{X} / \bar{\perp}] \in C$ Term so it must happen that $F V\left(t^{\prime}\right) \cap \bar{X}=\emptyset$ and therefore $t^{\prime} \equiv t^{\prime}[\bar{X} / \bar{\perp}] \equiv t$. But then let $\bar{X}=\bar{a}$ in $t^{\prime} \rightarrow^{l^{*}} t^{\prime} \equiv t$ by zero or more steps of (Elim), so $e \rightarrow^{l^{*}}$ let $\bar{X}=\bar{a}$ in $t^{\prime} \rightarrow^{l^{*}} t$, that is $e \rightarrow^{l^{*}} t$.

Finally, parts ii) and $i v$ ) follow from ii), iii) and Theorem 4.

As an immediate corollary of this completeness result and soundness (Theorem 10, we obtain the following result relating let-rewriting to CRWL and $\mathrm{CRWL}_{l e t}$ for total c-terms, which gives a clean and easy way to understand the formulation of the adequacy of let-rewriting. 
Corollary 1 (Equivalence of $C R W L_{l e t}$ and let-rewriting for total values)

For any program $\mathcal{P}, e \in L E x p$, and $t \in C$ Term we have

$$
\mathcal{P} \vdash_{C R W L_{l e t}} e \rightarrow t \text { iff } e \rightarrow^{l *} t .
$$

Besides if neither $\mathcal{P}$ nor $e$ have lets then we also have

$$
\mathcal{P} \vdash_{C R W L} e \rightarrow t \text { iff } e \rightarrow^{l *} t .
$$

As final consequence of Theorems 10 and 11 we obtain another strong equivalence result for both formalisms, this time expressed in terms of semantics and hypersemantics.

Theorem 12 (Equivalence of $C R W L_{\text {let }}$ and let-rewriting)

For any program $\mathcal{P}$ and $e \in L$ Exp:

i) $\llbracket e \rrbracket=\left\{\left|e^{\prime}\right| \mid e \rightarrow^{l^{*}} e^{\prime}\right\} \downarrow$

ii) $\llbracket e \rrbracket=\lambda \theta \in C S u b s t_{\perp} \cdot\left(\left\{\left|e^{\prime}\right| \mid e \rightarrow^{l^{*}} e^{\prime}\right\} \downarrow\right)$

where $\downarrow$ is the downward closure operator defined in Lemma 5

Proof

i) We prove both inclusions. Regarding $\llbracket e \rrbracket \subseteq\left\{\left|e^{\prime}\right| \mid e \rightarrow^{l^{*}} e^{\prime}\right\} \downarrow$, assume $t \in \llbracket e \rrbracket$. By Theorem 11 there must exist some $e^{\prime} \in \operatorname{LExp}$ such that $e \rightarrow^{l^{*}} e^{\prime}$ and $t \sqsubseteq\left|e^{\prime}\right|$, therefore $\left|e^{\prime}\right| \in\left\{\left|e^{\prime}\right| \mid e \rightarrow^{l^{*}} e^{\prime}\right\}$. But this, combined with $t \sqsubseteq\left|e^{\prime}\right|$, results in $t \in\left\{\left|e^{\prime}\right| \mid e \rightarrow^{l^{*}} e^{\prime}\right\} \downarrow$.

Regarding the other inclusion, consider some $t \in\left\{\left|e^{\prime}\right| \mid e \rightarrow^{l^{*}} e^{\prime}\right\} \downarrow$. By definition of the $\downarrow$ operator, there must exist some $e^{\prime} \in L \operatorname{Exp}$ such that $t \sqsubseteq\left|e^{\prime}\right|$ and $e \rightarrow^{l^{*}} e^{\prime}$. But that implies $\left|e^{\prime}\right| \in \llbracket e \rrbracket$, by Theorem 10 , which combined with $t \sqsubseteq\left|e^{\prime}\right|$ and the polarity property (Proposition 3) gives us that $t \in \llbracket e \rrbracket$.

ii) Trivial by applying the previous item and the definition of hypersemantics of an expression.

\section{Semantic reasoning}

Having equivalent notions of semantics and reduction allows to reason interchangeably at the rewriting and semantic levels. In this section we show the power of such technique in different situations. We start with a concrete example, adapted from (López-Fraguas et al. 2009b), where semantic reasoning leads easily to conclusions non-trivially achievable when thinking directly in operational terms.

\section{Example 4}

Imagine a program using constructors $a, b \in C S^{0}, c \in C S^{1}, d \in C S^{2}$ and defining a function $f \in F S^{1}$ for which we know that $f(a)$ can be let-rewritten to $c(a)$ and $c(b)$ but no other c-terms. Consider also an expression $e$ having $f(a)$ as subexpression, i.e., $e$ has the shape $\mathcal{C}[f(a)]$. We are interested now in the following question: can we safely replace in $e$ the subexpression $f(a)$ by any other ground expression $e^{\prime}$ 
let-reducible to the same set of values ${ }^{3}$ ? By safely we mean not changing the values reachable from $e$.

The question is less trivial than it could appear. For instance, if reductions were made with term rewriting instead of let-rewriting -i.e., considering run-time instead of call-time choice - the answer is negative (López-Fraguas et al. 2009b). To see that, consider the program

$$
\begin{array}{lll}
f(a) \rightarrow c(a) & g \rightarrow a & h(c(X)) \rightarrow d(X, X) \\
f(a) \rightarrow c(b) & g \rightarrow b
\end{array}
$$

and the expressions $e \equiv h(f(a))$ and $e^{\prime} \equiv c(g)$. All this is compatible with the assumptions of our problem. However, $e$ is reducible by term rewriting only to $d(a, a)$ and $d(b, b)$, while replacing $f(a)$ by $e^{\prime}$ in $e$ gives $h(c(g))$, which is reducible by term rewriting to two additional values, $d(a, b)$ and $d(b, a)$; thus, the replacement of $f(a)$ by $e^{\prime}$ has been unsafe.

However, the answer to our question is affirmative in general for let-rewriting, as it is very easily proved by a semantic reasoning using compositionality of $\mathrm{CRWL}_{l e t}$ : the assumption on $f(a)$ and $e^{\prime}$ means that they have the same denotation $\llbracket f(a) \rrbracket=$ $\llbracket e^{\prime} \rrbracket=\{c(a), c(b)\} \downarrow$ and, since they are ground, the same hyperdenotation $\llbracket f(a) \rrbracket=$ $\llbracket e^{\prime} \rrbracket=\lambda \theta .\{c(a), c(b)\} \downarrow$. By compositionality of hypersemantics, $\mathcal{C}[f(a)]$ and $\mathcal{C}\left[e^{\prime}\right]$ have the same (hyper)denotation, too. By equivalence of $\mathrm{CRWL}_{l e t}$ and let-rewriting this implies that both expressions reach the same value by let-rewriting.

Despite its simplicity, the example raises naturally interesting questions about replaceability, for which semantic methods could be simpler than direct reasonings about reduction sequences. This is connected to the full abstraction problem that we have investigated for run-time and call-time choice in (López-Fraguas et al. 2009b; López-Fraguas and Rodríguez-Hortalá 2010).

Semantic methods can be also used to prove the correctness of new operational rules not directly provided by our set of let-rewriting rules. Such rules can be useful for different purposes: to make computations simpler, for program transformations, to obtain new properties of the framework, ... Consider for instance the following generalization of the (LetIn) rule in Figure 5

(CLetIn) $\mathcal{C}[e] \rightarrow^{l}$ let $X=e$ in $\mathcal{C}[X], \quad$ if $B V(\mathcal{C}) \cap F V(e)=\emptyset$ and $X$ is fresh

This rule allows to create let-bindings in more situations and to put them in outer positions than the original (LetIn) rule. If we have not considered it in the definition of let-rewriting is because it would destroy the strong termination property of Proposition 2, as it is easy to see. However, this rule may shorten derivations. For instance, the derivation in Example 2 could be shortened to:

${ }^{3}$ More precisely, to the same set of shells in the sense of Theorem 12 part $i$ ). 


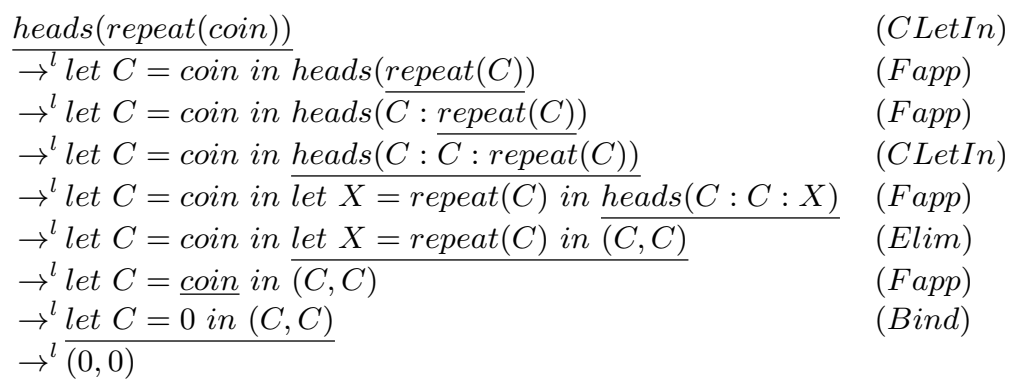

Reasoning the correctness of (CLetIn) rule is not difficult by means of semantic methods. We only need to prove that the rule preserves hypersemantics.

Lemma 9

If $B V(\mathcal{C}) \cap F V(e)=\emptyset$ and $X$ is fresh, then $\llbracket \mathcal{C}[e] \rrbracket=\llbracket$ let $X=e$ in $\mathcal{C}[X] \rrbracket$.

Proof

Assume an arbitrary $\theta \in C$ Subst $t_{\perp}$ :

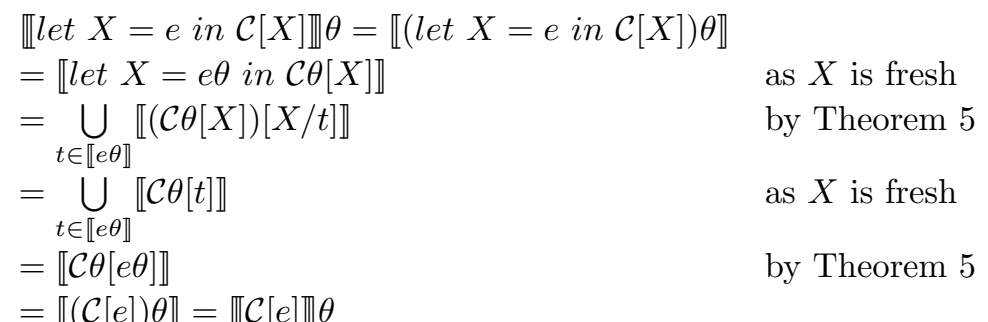

The rule (CLetIn) is indeed used in some of the proofs in the online appendix, together with another derived rule:

(Dist) $\mathcal{C}\left[\right.$ let $X=e_{1}$ in $\left.e_{2}\right] \rightarrow^{l}$ let $X=e_{1}$ in $\mathcal{C}\left[e_{2}\right]$, if $B V(\mathcal{C}) \cap F V\left(e_{1}\right)=\emptyset$ and $X \notin F V(\mathcal{C})$

which also preserves hypersemantics:

Lemma 10

If $B V(\mathcal{C}) \cap F V\left(e_{1}\right)=\emptyset$ and $X \notin F V(\mathcal{C})$ then $\llbracket \mathcal{C}\left[\right.$ let $X=e_{1}$ in $\left.e_{2}\right] \rrbracket=\llbracket l$ let $X=$ $e_{1}$ in $\mathcal{C}\left[e_{2}\right] \rrbracket$.

These ideas can be made more general. Consider the equivalence relation $e_{1} \asymp$ $e_{2}$ iff $\llbracket e_{1} \rrbracket=\llbracket e_{2} \rrbracket$. This relation is especially relevant because $e_{1} \asymp e_{2}$ iff $\forall \mathcal{C} \in$ Cntxt. $\llbracket \mathcal{C}[e] \rrbracket=\llbracket \mathcal{C}\left[e^{\prime}\right] \rrbracket$, by Theorem 6 . We can contemplate $\asymp$ as an abstract, although non-effective, reduction relation, of which the relations $\rightarrow^{\operatorname{lnf}}$ of Section 4 and the rules (CLetIn) and (Dist) are particular subrelations. It is trivial to check that, by construction, the combined relation $\rightarrow^{l} \cup \asymp$ is sound and complete wrt. $\mathrm{CRWL}_{\text {let }}$. We can use that relation to reason about the meaning or equivalence of let-expressions and programs. We could also employ it in the definition of ondemand evaluation strategies for let-rewriting. As any subrelation of $\rightarrow^{l} \cup \asymp$ is 
sound wrt. $\mathrm{CRWL}_{l e t}$, an approach to strategies for let-rewriting could consist in defining a suitable operationally effective subrelation of $\rightarrow^{l} \cup \asymp$ and then proving its completeness and optimality (if it is the case).

\subsection{A case study: correctness of bubbling}

We develop here another nice application of the 'semantic route', where let-rewriting provides a good level of abstraction to formulate a new operational rule - bubbling while the semantic point of view is appropriate for proving its correctness.

Bubbling, proposed in (Antoy et al. 2007), is an operational rule devised to improve the efficiency of functional logic computations. Its correctness was formally studied in (Antoy et al. 2006) in the framework of a variant (Echahed and Janodet 1998) of term graph rewriting. The idea of bubbling is to concentrate all nondeterminism of a system into a choice operation ? defined by the rules $X$ ? $Y \rightarrow X$ and $X$ ? $Y \rightarrow Y$, and to lift applications of ? out of their surrounding context, as illustrated by the following graph transformation taken from (Antoy et al. 2006):

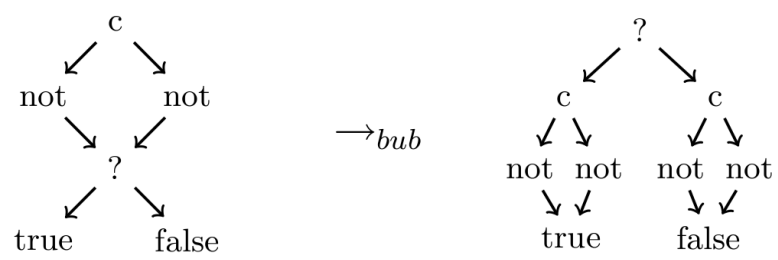

As it is shown in (Antoy et al. 2007), bubbling can be implemented in such a way that many functional logic programs become more efficient, but we will not deal with these issues here.

Due to the technical particularities of term graph rewriting, not only the proof of correctness, but even the definition of bubbling in (Antoy et al. 2007, Antoy et al. 2006) are involved and need subtle care concerning the appropriate contexts over which choices can be bubbled. In contrast, bubbling can be expressed within our framework in a remarkably easy and abstract way as a new rewriting rule:

(Bub) $\mathcal{C}\left[e_{1} ? e_{2}\right] \rightarrow{ }^{b u b} \mathcal{C}\left[e_{1}\right] ? \mathcal{C}\left[e_{2}\right]$, for $e_{1}, e_{2} \in L E x p$

With this rule, the bubbling step corresponding to the graph transformation of the example above is:

$$
\begin{aligned}
& \text { let } X=\text { true } ? \text { false in } c(n o t(X), \operatorname{not}(X)) \rightarrow^{\text {bub }} \\
& \text { let } X=\text { true in } c(\operatorname{not}(X), \operatorname{not}(X)) ? \text { let } X=\text { false in } c(\operatorname{not}(X), \operatorname{not}(X))
\end{aligned}
$$

Notice that the effect of this bubbling step is not a shortening of any existing letrewriting derivation; bubbling is indeed a genuine new rule, the correctness of which must be therefore subject of proof. Call-time choice is essential, since bubbling is not correct with respect to ordinary term rewriting, i.e., run-time choice.

Counterexample 2 (Incorrectness of bubbling for run-time choice)

Consider a function pair defined by the rule $\operatorname{pair}(X) \rightarrow c(X, X)$ and the expression 
$\operatorname{pair}\left(0\right.$ ? 1) for $c \in C S^{2}$ and $0,1 \in C S^{0}$. Under term rewriting/run-time choice the derivation

$$
\operatorname{pair}(0 ? 1) \rightarrow c(0 ? 1,0 ? 1) \rightarrow c(0,0 ? 1) \rightarrow c(0,1)
$$

is valid. But if we performed the bubbling step

$$
\operatorname{pair}(0 ? 1) \rightarrow^{\text {bub }} \operatorname{pair}(0) ? \operatorname{pair}(1)
$$

then the c-term $c(0,1)$ would not be reachable anymore by term rewriting from pair(0) ? pair(1).

Formulating and proving the correctness of bubbling for call-time choice becomes easy by using semantics. As we did before, we simply prove that bubbling steps preserve hypersemantics. We need first a basic property of the (hyper)semantics of binary choice ?. Its proof stems almost immediately from the rules for ? and the definition of CRWL-(hyper)denotation.

Proposition 9 ((Hyper)semantic properties of ?)

For any $e_{1}, e_{2} \in L E x p_{\perp}$

i) $\llbracket e_{1} ? e_{2} \rrbracket=\llbracket e_{1} \rrbracket \cup \llbracket e_{2} \rrbracket$

ii) $\llbracket e_{1} ? e_{2} \rrbracket=\llbracket e_{1} \rrbracket \amalg \llbracket e_{2} \rrbracket$

Combining this property with some of the powerful hypersemantic results from Section 4.2 leads to an appealing proof of the correctness of bubbling.

Theorem 13 (Correctness of bubbling for call-time choice)

If $e \rightarrow^{b u b} e^{\prime}$ then $\llbracket e \rrbracket=\llbracket e^{\prime} \rrbracket$, for any $e, e^{\prime} \in L \operatorname{Exp}$.

Proof

If $e \rightarrow^{b u b} e^{\prime}$ then $e=\mathcal{C}\left[e_{1} ? e_{2}\right]$ and $e^{\prime}=\mathcal{C}\left[e_{1}\right] ? \mathcal{C}\left[e_{2}\right]$, for some $e_{1}, e_{2}$. Then:

$$
\begin{aligned}
& \llbracket \mathcal{C}\left[e_{1} ? e_{2}\right] \rrbracket=\llbracket \mathcal{C} \rrbracket \llbracket e_{1} ? e_{2} \rrbracket \quad \text { by Theorem } 6 \\
& =\llbracket \mathcal{C} \rrbracket\left(\llbracket e_{1} \rrbracket \llbracket \llbracket e_{2} \rrbracket\right) \quad \text { by Proposition } 9 \text { ii) } \\
& =\llbracket \mathcal{C} \rrbracket \llbracket e_{1} \rrbracket \amalg \llbracket \mathcal{C} \rrbracket \llbracket e_{2} \rrbracket \quad \text { by Proposition } 7 \\
& =\llbracket \mathcal{C}\left[e_{1}\right] \rrbracket \amalg \llbracket \mathcal{C}\left[e_{2}\right] \rrbracket \quad \text { by Theorem } 6 \\
& =\llbracket \mathcal{C}\left[e_{1}\right] ? \mathcal{C}\left[e_{2}\right] \rrbracket \quad \text { by Proposition } 9 \text { ii) }
\end{aligned}
$$

This property was proved also for the HO case in (López-Fraguas et al. 2008). But the proof given here is much more elegant thanks to the new semantic tools developed in Section 4.2

\section{Let-narrowing}

It is well known that there are situations in functional logic computations where rewriting is not enough and must be lifted to some kind of narrowing, because the expression being reduced contains variables for which different bindings might produce different evaluation results. This might happen either because variables are 
already present in the initial expression to reduce, or due to the presence of extra variables in the program rules. In the latter case let-rewriting certainly works, but not in an effective way, since the parameter passing substitution in the rule (Fapp) of Figure 5 (page 16) 'magically' guesses the appropriate values for those extra variables (see Example 6 below). Some works (Antoy and Hanus 2006; Dios-Castro and López-Fraguas 2007; Braßel and Huch 2007) have proved that guessing can be replaced by a systematic non-deterministic generation of all (ground) possible values. However, this does not cover all aspects of narrowing, which is able to produce non-ground answers, while generator functions are not. In this section we present let-narrowing, a natural lifting of let-rewriting devised to effectively deal with free and extra variables.

Using the notation of contexts, the standard definition of narrowing as a lifting of term rewriting in ordinary TRS's is the following: $\mathcal{C}[f(\bar{t})] \rightsquigarrow_{\theta} \mathcal{C} \theta[r \theta]$, if $\theta$ is a mgu of $f(\bar{t})$ and $f(\bar{s})$, where $f(\bar{s}) \rightarrow r$ is a fresh variant of a rule of the TRS. The requirement that the binding substitution $\theta$ is a mgu can be relaxed to accomplish with certain narrowing strategies like needed narrowing (Antoy et al. 2000), which use unifiers but not necessarily most general ones.

This definition of narrowing cannot be directly translated as it is to the case of letrewriting, for two reasons. First, binding substitutions must be c-substitutions, as for the case of let-rewriting. Second, let-bound variables should not be narrowed, but their values should be rather obtained by evaluation of their binding expressions. The following example illustrates some of the points above.

Example 5

Consider the following program over Peano natural numbers:

$$
\begin{array}{ll}
0+Y \rightarrow Y & \text { even }(X) \rightarrow \text { if }(Y+Y==X) \text { then true } \\
s(X)+Y \rightarrow s(X+Y) & \text { if true then } Y \rightarrow Y \\
0==0 \rightarrow \text { true } & s(X)==s(Y) \rightarrow X==Y \\
0==s(Y) \rightarrow \text { false } & s(X)==0 \rightarrow \text { false } \\
\text { coin } \rightarrow 0 & \text { coin } \rightarrow s(0)
\end{array}
$$

Notice the extra variable $Y$ in the rule for even. The evaluation of even(coin) by let-rewriting could start as follows:

$$
\begin{aligned}
& \text { even }(\text { coin }) \rightarrow^{l} \text { let } X=\text { coin in even }(X) \\
& \rightarrow^{l} \text { let } X=\text { coin in if }(Y+Y==X) \text { then true } \\
& \rightarrow^{l} \text { let } X=\text { coin in let } U=Y+Y \text { in let } V=(U==X) \text { in if } V \text { then true } \\
& \rightarrow^{l} \text { let } U=Y+Y \text { in let } V=(U==0) \text { in if } V \text { then true }
\end{aligned}
$$

Now, because all function applications involve variables, the evaluation cannot continue merely by rewriting, and therefore narrowing is required instead. We should not perform standard narrowing steps that bind already let-bound variables; otherwise, the syntax of let-expressions can be lost. For instance, narrowing at if $V$ then true generates the binding $[V /$ true $]$ that, if applied naively to the surrounding context, results in the syntactically illegal expression:

$$
\text { let } U=Y+Y \text { in let true }=(U==0) \text { in true }
$$


(X) $e \rightsquigarrow_{\epsilon}^{l} e^{\prime} \quad$ if $e \rightarrow^{l} e^{\prime}$ using $\boldsymbol{X} \in\{$ LetIn, Bind, Elim, Flat $\}$ in Figure 5 (page 16).

(Narr) $f(\bar{t}) \rightsquigarrow_{\theta}^{l} r \theta$, for any fresh variant $(f(\bar{p}) \rightarrow r) \in \mathcal{P}$ and $\theta \in C$ Subst such that $f(\bar{t}) \theta \equiv f(\bar{p}) \theta$.

(Contx) $\mathcal{C}[e] \rightsquigarrow_{\theta}^{l} \mathcal{C} \theta\left[e^{\prime}\right]$, for $\mathcal{C} \neq[]$, if $e \rightsquigarrow^{l}{ }_{\theta} e^{\prime}$ by any of the previous rules, and if the step is (Narr) using $(f(\bar{p}) \rightarrow r) \in \mathcal{P}$, then:

$$
\begin{array}{ll}
\text { (i) } & \operatorname{dom}(\theta) \cap B V(\mathcal{C})=\emptyset \\
\text { (ii) } & \operatorname{vRan}\left(\left.\theta\right|_{\backslash \operatorname{var}(\bar{p})}\right) \cap B V(\mathcal{C})=\emptyset
\end{array}
$$

Fig. 6. Rules of the let-narrowing relation $\rightsquigarrow^{l}$

What is harmless is to perform narrowing at $Y+Y$ ( $Y$ is a free variable). This gives the substitution $[Y / 0]$ and the result 0 for the subexpression $Y+Y$. Placing it in its surrounding context, the derivation continues as follows:

$$
\begin{aligned}
& \text { let } U=0 \text { in let } V=(U==0) \text { in if } V \text { then true } \\
& \rightarrow^{l} \text { let } V=(0==0) \text { in if } V \text { then true } \\
& \rightarrow^{l} \text { let } V=\text { true in if } V \text { then true } \\
& \rightarrow^{l} \text { if true then true } \rightarrow^{l} \text { true }
\end{aligned}
$$

The previous example shows that let-narrowing must protect bound variables against substitutions, which is the key observation for defining narrowing in presence of let-bindings.

The one-step let-narrowing relation $e \rightsquigarrow_{\theta}^{l} e^{\prime}$ (assuming a given program $\mathcal{P}$ ) is defined in Figure6.

- The rule (X) collects (Elim), (Bind), (Flat), (LetIn) of $\rightarrow^{l}$, that remain the same in $\rightsquigarrow^{l}$, except for the decoration with the empty substitution $\epsilon$.

- The rule (Narr) performs a narrowing step in a proper sense. To avoid unnecessary loss of generality or applicability of our approach, we do not impose $\theta$ to be a mgu. For the sake of readability, we will sometimes decorate (Narr) steps with $\left.\theta\right|_{F V(f(\bar{t}))}$ instead of $\theta$, i.e., with the projection over the variables in the narrowed expression.

- The rule (Contx) indicates how to use the previous rules in inner positions. The condition $\mathcal{C} \neq[$ ] simply avoids trivial overlappings of (Contx) with the previous rules. The rest of the conditions are set to ensure that the combination of (Contx) with (Narr) makes a proper treatment of bound variables:

- (i) expresses the protection of bound variables against narrowing justified in Example 5

- (ii) is a rather technical condition needed to prevent undesired situations when the narrowing step has used a program rule with extra variables and a unifier $\theta$ which is not a mgu. Concretely, the condition states that the bindings created by $\theta$ for the extra variables in the program rule do not introduce variables that are bound by the surrounding context $\mathcal{C}$. To see the problems that can arise without (ii), consider for instance the program rules $f \rightarrow Y$ and loop $\rightarrow$ loop and the expression let $X=$ loop in $f$. A legal reduction for this expression, respecting condition (ii) could be the 
following:

$$
\text { let } X=\text { loop in } f \rightsquigarrow^{l}{ }_{\epsilon} \text { let } X=\text { loop in } Z
$$

by applying (Narr) to $f$ with $\theta=\epsilon$ taking the fresh variant rule $f \rightarrow Z$, and using (Contx) for the whole expression. However, if we drop condition (ii) we could perform a similar derivation using the same fresh variant of the rule for $f$, but now using the substitution $\theta=[Z / X]$ :

$$
\text { let } X=\text { loop in } f \rightsquigarrow^{l}{ }_{\epsilon} \text { let } X=\text { loop in } X
$$

which is certainly not intended because the free variable $Z$ in the previous derivation appears now as a bound variable, i.e., we get an undesired capture of variables.

We remark that if the substitution $\theta$ in (Narr) is chosen to be a standard mgd ${ }^{4}$ of $f(\bar{t})$ and $f(\bar{p})$ (which is always possible) then the condition (ii) is always fulfilled.

The one-step relation $\rightsquigarrow_{\theta}^{l}$ is extended in the natural way to the multiple-steps narrowing relation $\rightsquigarrow l_{\theta}^{*}$, which is defined as the least relation verifying:

$$
e \rightsquigarrow l_{\epsilon}^{l^{*}} e \quad e \rightsquigarrow{ }_{\theta_{1}}^{l} e_{1} \rightsquigarrow{ }_{\theta_{2}}^{l} \ldots e_{n} \rightsquigarrow_{\theta_{n}}^{l} e^{\prime} \Rightarrow e \rightsquigarrow_{\theta_{1} \ldots \theta_{n}}^{l^{*}} e^{\prime}
$$

We write $e \rightsquigarrow_{\theta}^{l^{n}} e^{\prime}$ for a n-steps narrowing sequence.

\section{Example 6}

Example 5 essentially contains already a narrowing derivation. For the sake of clarity, we repeat it here making explicit the rule of let-narrowing used at each step (maybe in combination with (Contx), which is not written). Besides, if the step uses (Narr), the narrowed expression is underlined.

$$
\begin{aligned}
& \text { even }(\operatorname{coin}) \rightsquigarrow^{l}{ }_{\epsilon} \\
& \text { let } X=\text { coin in even }(X) \rightsquigarrow^{l}{ }_{\epsilon} \\
& \text { (LetIn) } \\
& \text { (Narr) } \\
& \text { let } X=\text { coin in } \overline{\text { if } Y+Y}==X \text { then true } \varliminf_{\epsilon}^{l^{3}} \\
& \text { (LetIn } \left.{ }^{2}, \text { Flat }\right) \\
& \text { let } X=\underline{\text { coin }} \text { in let } U=Y+Y \text { in } \\
& \text { let } \overline{V=}(U==X) \text { in if } V \text { then true } \rightsquigarrow^{l} \epsilon \\
& \text { let } X=0 \text { in let } U=Y+Y \text { in } \\
& \text { let } V=(U==X) \text { in if } V \text { then true } \rightsquigarrow^{l} \epsilon \\
& (N a r r) \\
& \text { (Bind) } \\
& \text { (Narr) } \\
& \text { (Bind) } \\
& \text { (Narr) } \\
& \text { (Bind) } \\
& \text { (Narr) } \\
& \begin{array}{l}
\text { let } U=Y+Y \text { in let } V=(U==0) \text { in if } V \text { then true } \rightsquigarrow^{l}[Y / 0] \\
\text { let } U=0 \text { in let } V=(U==0) \text { in if } V \text { then true } \rightsquigarrow^{l}{ }^{6}
\end{array} \\
& \text { let } V=(0==0) \text { in if } V \text { then true } \rightsquigarrow^{l} \epsilon
\end{aligned}
$$

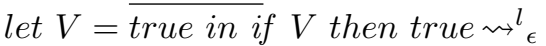

$$
\begin{aligned}
& \text { if true then true } \rightsquigarrow^{l}{ }_{\epsilon} \\
& \text { true }
\end{aligned}
$$

Notice that all (Narr) steps in the derivation except one have $\epsilon$ as narrowing substitution (because of the projection over the variables of the narrowed expression), so they are really rewriting steps. An additional remark that could help to

\footnotetext{
${ }^{4}$ By standard mgu of $t, s$ we mean an idempotent mgu $\theta$ with $\operatorname{dom}(\theta) \cup \operatorname{ran}(\theta) \subseteq \operatorname{var}(t) \cup \operatorname{var}(s)$.
} 
further explain the relationship between the let-narrowing relation $\rightsquigarrow^{l}$ and the letrewriting relation $\rightarrow^{l}$ is the following: since we have even (coin) $\rightsquigarrow_{\theta}^{l}$ true for some $\theta$, but even(coin) is ground, Theorem 14 in next section ensures that there must be also a successful let-rewriting derivation even $(\operatorname{coin}) \rightarrow^{l^{*}} \operatorname{true}$. This derivation could have the form:

$$
\begin{array}{ll}
\text { even }(\operatorname{coin}) \rightarrow^{l} & (\text { LetIn }) \\
\text { let } X=\text { coin in even }(X) \rightarrow^{l} & (\text { Fapp }) \\
\text { let } X=\text { coin in if }(0+0==X) \text { then true } \rightarrow^{l} & \\
\ldots \ldots \ldots \rightarrow^{l} \text { true } &
\end{array}
$$

The indicated (Fapp)-step in this let-rewriting derivation has used the substitution $[Y / 0]$, thus anticipating and 'magically guessing' the correct value of the extra variable $Y$ of the rule of even. In contrast, in the let-narrowing derivation the binding for $Y$ is not done while reducing even $(X)$ but in a later (Narr)-step over $Y+Y$. This corresponds closely to the behavior of narrowing-based systems like Toy or Curry.

\subsection{Soundness and completeness of the let-narrowing relation $\rightsquigarrow^{l}$}

In this section we show the adequacy of let-narrowing wrt. let-rewriting. From now on we assume a fixed program $\mathcal{P}$.

As usual with narrowing relations, soundness results are not difficult to formulate and prove. The following soundness result for $\rightsquigarrow^{l}$ states that we can mimic any $\rightsquigarrow^{l}$ derivation with $\rightarrow^{l}$ by applying over the starting expression the substitution computed by the original let-narrowing derivation.

Theorem 14 (Soundness of the let-narrowing relation $\rightsquigarrow^{l}$ )

For any $e, e^{\prime} \in L \operatorname{Exp}, e \rightsquigarrow{ }_{\theta}^{l^{*}} e^{\prime}$ implies $e \theta \rightarrow^{l^{*}} e^{\prime}$.

Completeness is more complicated to prove. The key result is a generalization to let-rewriting of Hullot's lifting lemma (Hullot 1980) for classical term rewriting and narrowing. It states that any rewrite sequence for a particular instance of an expression can be generalized by a narrowing derivation.

Lemma 11 (Lifting lemma for the let-rewriting relation $\rightarrow^{l}$ )

Let $e, e^{\prime} \in L \operatorname{Exp}$ such that $e \theta \rightarrow^{l^{*}} e^{\prime}$ for some $\theta \in C S u b s t$, and let $\mathcal{W}, \mathcal{B} \subseteq \mathcal{V}$ with $\operatorname{dom}(\theta) \cup F V(e) \subseteq \mathcal{W}, B V(e) \subseteq \mathcal{B}$ and $(\operatorname{dom}(\theta) \cup \operatorname{vran}(\theta)) \cap \mathcal{B}=\emptyset$, and for each (Fapp) step of $e \theta \rightarrow^{l^{*}} e^{\prime}$ using a rule $R \in \mathcal{P}$ and a substitution $\gamma \in C$ Subst then $\operatorname{vran}\left(\left.\gamma\right|_{v E x t r a(R)}\right) \cap \mathcal{B}=\emptyset$. Then there exist a derivation $e{ }_{\sigma}^{l^{*}} e^{\prime \prime}$ and $\theta^{\prime} \in C S u b s t$ such that:

$$
\text { (i) } e^{\prime \prime} \theta^{\prime}=e^{\prime} \quad \text { (ii) } \sigma \theta^{\prime}=\theta[\mathcal{W}] \quad \text { (iii) }\left(\operatorname{dom}\left(\theta^{\prime}\right) \cup \operatorname{vran}\left(\theta^{\prime}\right)\right) \cap \mathcal{B}=\emptyset
$$

Besides, the let-narrowing derivation can be chosen to use mgu's at each (Narr) step. Graphically: 


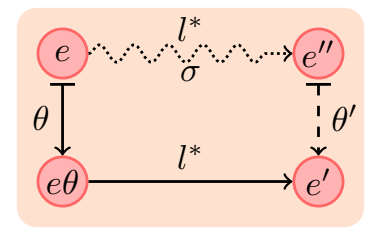

With the aid of this lemma we are now ready to state and prove the following strong completeness result for $\rightsquigarrow^{l}$.

Theorem 15 (Completeness of the let-narrowing relation $\rightsquigarrow^{l}$ )

Let $e, e^{\prime} \in L \operatorname{Exp}$ and $\theta \in C$ Subst. If $e \theta \rightarrow^{l *} e^{\prime}$, then there exist a let-narrowing derivation $e \rightsquigarrow{ }_{\sigma}^{*} e^{\prime \prime}$ and $\theta^{\prime} \in C S u b s t$ such that $e^{\prime \prime} \theta^{\prime} \equiv e^{\prime}$ and $\sigma \theta^{\prime}=\theta[F V(e)]$.

Proof

Applying Lemma 11 to $\left.e \theta\right|_{F V(e)} \rightarrow^{l^{*}} e^{\prime}$ with $\mathcal{W}=F V(e)$ and $\mathcal{B}=B V(e)$, as $\left.e \theta\right|_{F V(e)} \equiv e \theta$ and the additional conditions over $\mathcal{B}$ hold by the variable convention.

Finally, by combining Theorems 14 and 15 , we obtain a strong adequacy theorem for let-narrowing with respect to let-rewriting.

Theorem 16 (Adequacy of the let-narrowing relation $\rightsquigarrow^{l}$ wrt. $\rightarrow^{l}$ )

Let $e, e_{1} \in L \operatorname{Exp}$ and $\theta \in C$ Subst, then:

$$
e \theta \rightarrow^{l^{*}} e_{1} \Leftrightarrow \quad \begin{aligned}
& \text { there exist a let-narrowing derivation } e \rightsquigarrow{ }_{\sigma}^{l^{*}} e_{2} \text { and } \\
& \text { some } \theta^{\prime} \in C \text { Subst such that } \sigma \theta^{\prime}=\theta[F V(e)], e_{2} \theta^{\prime} \equiv e_{1}
\end{aligned}
$$

Proof

$(\Rightarrow)$ Assume $e \theta \rightarrow^{l^{*}} e_{1}$. As $\left.e \theta\right|_{F V(e)} \equiv e \theta$ then trivially $\left.e \theta\right|_{F V(e)} \rightarrow^{l *} e_{1}$. We can apply Lemma 11 taking $\mathcal{W}=F V(e)$ to get $e \rightsquigarrow{ }_{\sigma}{ }^{*} e_{2}$ such that there exists $\theta^{\prime} \in C S u b s t$ with $\sigma \theta^{\prime}=\left.\theta\right|_{F V(e)}[\mathcal{W}]$ and $e_{2} \theta^{\prime} \equiv e_{1}$. But as $\mathcal{W}=F V(e)$ then $\sigma \theta^{\prime}=\left.\theta\right|_{F V(e)}[\mathcal{W}]$ implies $\sigma \theta^{\prime}=\theta[F V(e)]$.

We remark that the lifting lemma ensures that the narrowing derivation can be chosen to use mgu's at each (Narr) step.

$(\Leftarrow)$ Assume $e \rightsquigarrow \frac{l^{*}}{\sigma} e_{2}$ and $\theta^{\prime}$ under the conditions above. Then by Theorem 14 we have $e \sigma \rightarrow^{l} e_{2}$. As $\rightarrow^{l}$ is closed under c-substitutions (Lemma 2) then $e \sigma \theta^{\prime} \rightarrow^{l *} e_{2} \theta^{\prime}$. But as $\sigma \theta^{\prime}=\theta[F V(e)]$, then $e \theta \equiv e \sigma \theta^{\prime} \rightarrow^{l *} e_{2} \theta^{\prime} \equiv e_{1}$.

\subsection{Organizing computations}

Deliberately, in this paper we have kept the definitions of let-rewriting and narrowing apart from any particular computation strategy. In this section we explain rather informally how the ideas of some known strategies for functional logic programming (Antoy 2005) can be adapted also to our formal setting. For the sake of brevity we focus only on let-narrowing computations. As a running example, consider the program 


$$
\begin{array}{ll}
\operatorname{leq}(0, Y) \rightarrow \text { true } & f(0) \rightarrow 0 \\
\text { leq }(s(X), 0) \rightarrow \text { false } & \\
\operatorname{leq}(s(X), s(Y)) \rightarrow \operatorname{leq}(X, Y) &
\end{array}
$$

and the initial expression $l e q(X, f(Y))$ to be let-narrowed using it.

As a first remark, when designing a strategy one can freely use 'peeling' steps in a don't care manner using the relation $\rightarrow^{\operatorname{lnf}}$ (Definition 3), since it is terminating and (hyper-)semantics-preserving. In our case one step suffices: $\operatorname{le} q(X, f(Y)){ }^{l}{ }_{\epsilon}$ let $U=$ $f(Y)$ in leq $(X, U)$. After a peeling (multi-)step, a (Narr) step must be done. Where? Certainly, the body $l e q(.$.$) must be narrowed at some point. One don't know pos-$ sibility is narrowing at $l e q(X, U)$ using the first rule for $l e q$ that does not bind $U$ : let $U=f(Y)$ in leq $(X, U) \rightsquigarrow_{[X / 0]}^{l}$ let $U=f(Y)$ in true. A new peeling step leads to a first final result true, with computed substitution $[X / 0]$.

The second and third rules for $l e q$ could lead to more results. Those rules have non-variable patterns as second arguments, and then the bound variable $U$ in leq $(X, U)$ inhibits a direct (Narr) step in that position. Typically it is said that $U$ is demanded by those leq rules. Therefore, we narrow $f(Y)$ to get values for $U$, and then we 'peel':

$$
\text { let } U=f(Y) \text { in } \operatorname{leq}(X, U) \rightsquigarrow_{[Y / 0]}^{l} \text { let } U=0 \text { in } \operatorname{leq}(X, U) \rightsquigarrow^{l}{ }_{\epsilon} \operatorname{leq}(X, 0)
$$

The computation proceeds now by two don't know choices using the rules for leq, leading to two more solutions (true, $[Y / 0, X / 0])$ and (false, $[Y / 0, X / s(Z)])$.

This implicitly applied strategy can be seen as a translation to let-narrowing of lazy narrowing (Moreno-Navarro and Rodríguez-Artalejo 1992; Alpuente et al. 2003). As a known drawback of lazy narrowing, notice that the second solution (true, $[Y / 0, X / 0])$ is redundant, since it is less general than the first one (true, $[X / 0])$. Redundancy is explained because we have narrowed the expression $f(Y)$ whose evaluation was demanded only by some of the rules for the outer function application leq $(X, f(Y))$, but after that we have used the rules not demanding the evaluation (the first rule for $l e q$ ). This problem is tackled successfully by needed narrowing Antoy et al. 2000) which takes into account, when narrowing an inner expression, what are the rules for an outer function application demanding such evaluation. A needed narrowing step 'anticipates' the substitution that will perform these rules when they are to be applied. The ideas of needed narrowing can be adapted to our setting. In our example, we get the following derivation instead of (1):

$$
\begin{aligned}
& \text { let } U=f(Y) \text { in leq }(X, U) \rightsquigarrow^{l}{ }_{[X / s(Z), Y / 0]} \text { let } U=0 \text { in leq }(s(Z), U) \rightsquigarrow^{l}{ }_{\epsilon} \\
& \text { leq }(s(Z), 0) \rightsquigarrow{ }_{\epsilon} \text { false }
\end{aligned}
$$

The first step does not use a mgu. This a typical feature of needed narrowing, and is also allowed by let-narrowing steps. Needed narrowing steps rely on definitional trees that structure demandness information from the rules of a given function. This information can be embedded also into a program transformation. There are simple transformations for which the transformed program, under a lazy narrowing regime using mgu's, obtains the same solutions than the original program (Zartmann 1997), although it is not guaranteed that the number of steps is also preserved. In our example, the definition of leq can be transformed as follows: 


$$
\begin{array}{ll}
\operatorname{leq}(0, Y) \rightarrow \text { true } & \operatorname{leq} S(X, 0) \rightarrow \text { false } \\
\operatorname{leq}(s(X), Y) \rightarrow \operatorname{leq} S(X, Y) & \operatorname{leq} S(X, s(Y)) \rightarrow \operatorname{leq}(X, Y)
\end{array}
$$

As happened with (1'), the derivation

$$
\begin{aligned}
& \text { let } U=f(Y) \text { in leq }(X, U) \rightsquigarrow^{l}[X / s(Z)] \text { let } U=f(Y) \text { in leq } S(Z, U) \rightsquigarrow^{l}[Y / 0] \\
& \text { let } U=0 \text { in leqS }(Z, U) \rightsquigarrow^{l}{ }_{\epsilon} \text { leq } S(Z, 0) \rightsquigarrow^{l}{ }_{\epsilon} \text { false }
\end{aligned}
$$

gets rid of redundant solutions.

To which extent do our results guarantee the adequateness of the adaptation to let-narrowing of these strategies or others that could be defined? Certainly any strategy is sound for call-time choice semantics, because unrestricted $\rightsquigarrow^{l}$ is already sound (Theorem 14). This will be true also if the strategy uses derived rules in the sense of Section 5. With respect to completeness, we know that the space of let-narrowing derivations is complete wrt. let-rewriting (Theorem 15). But this does not imply the completeness of the strategy, which in general will determine a smaller narrowing space. Therefore completeness of the strategy must be proved independently. Such a proof may use semantic methods (i.e., prove completeness wrt. CRWL-semantics) or operational methods (i.e., prove completeness wrt. $\rightarrow^{l}-$ derivations). We will not go deeper into the issue of strategies.

\section{Let-rewriting versus classical term rewriting}

In this section we examine the relationship between let-rewriting and ordinary term rewriting, with the focus put in the set of c-terms reachable by rewriting with each of these relations. As term rewriting is not able to handle expressions with letbindings, during this section we assume that all considered programs do not have let-bindings in the right-hand side of its rules.

We will first prove in Section 7.1 that let-rewriting is sound with respect to term rewriting, in the sense that any c-term that can we reached by a let-rewriting derivation from a given expression can also be reached by a term rewriting derivation starting from the same expression. As we know, completeness does not hold in general because run-time choice computes more values than call-time choice for arbitrary programs. However, we will be able to prove completeness of let-rewriting wrt. term rewriting for the class of deterministic programs, a notion close to confluence that will be defined in Section 7.2. Finally, we will conclude in Section 7.3 with a comparison between let-narrowing and narrowing, that will follow easily from the results in previous subsections and the adequacy of let-narrowing to let-rewriting.

Thanks to the strong equivalence between CRWL and let-rewriting we can choose the most appropriate point of view for each of the two goals (soundness and completeness): we will use let-rewriting for proving soundness, and CRWL for defining the property of determinism and proving that, under determinism, completeness of let-rewriting wrt. term rewriting also holds.

\subsection{Soundness of let-rewriting wrt. classical term rewriting}

In order to relate let-rewriting to term rewriting, we first need to find a way for term rewriting to cope with let-bindings, which are not supported by its syntax, 
that is only able to handle expressions from Exp. Therefore, we define the following syntactic transformation from LExp into Exp that takes care of removing the let constructions, thus losing the sharing information they provide.

Definition 9 (Let-binding elimination transformation)

Given $e \in L E x p$ we define its transformation into a let-free expression $\widehat{e} \in \operatorname{Exp}$ as:

$$
\begin{aligned}
& \widehat{X}={ }_{\operatorname{def} X} \\
& h\left(e_{1}, \ldots, e_{n}\right)=\operatorname{def}_{\operatorname{def}} h\left(\widehat{e_{1}}, \ldots, \widehat{e_{n}}\right) \\
& \text { let } \widehat{X=e_{1}} \text { in } e_{2}={ }_{\operatorname{def}} \widehat{e_{2}}\left[X / \widehat{e_{1}}\right]
\end{aligned}
$$

Note that $\widehat{e} \equiv e$ for any $e \in \operatorname{Exp}$.

We will need also the following auxiliary lemma showing the interaction between term rewriting derivations and substitution application.

Lemma 12 (Copy lemma)

For all $e, e_{1}, e_{2} \in \operatorname{Exp}, X \in \mathcal{V}$ :

i) $e_{1} \rightarrow e_{2}$ implies $e\left[X / e_{1}\right] \rightarrow^{*} e\left[X / e_{2}\right]$.

ii) $e_{1} \rightarrow^{*} e_{2}$ implies $e\left[X / e_{1}\right] \rightarrow^{*} e\left[X / e_{2}\right]$.

Note how in $i$ ), each of the different copies of $e_{1}$ introduced in $e$ by the substitution has to be reduced to $e_{2}$ in a different term rewriting step in order to reach the expression $e\left[X / e_{2}\right]$.

Using this lemma we can get a first soundness result stating that the result of one let-rewriting step can also be obtained in zero or more steps of ordinary rewriting, after erasing the sharing information by means of the let-binding elimination transformation.

Lemma 13 (One-Step Soundness of let-rewriting wrt. term rewriting)

For all $e, e^{\prime} \in L \operatorname{Exp}$ we have that $e \rightarrow^{l} e^{\prime}$ implies $\widehat{e} \rightarrow{ }^{*} \widehat{e}^{\prime}$.

The remaining soundness results follow easily from this lemma. The first one shows how we can mimic let-rewriting with term rewriting through the let-binding elimination transformation. But then, as $\widehat{e} \equiv e$ for any $e \in E x p$, we conclude that for let-free expressions let-rewriting is a subrelation of term rewriting.

Theorem 17 (Soundness of let-rewriting wrt. term rewriting)

For any $e, e^{\prime} \in L \operatorname{Exp}$ we have that $e \rightarrow^{l^{*}} e^{\prime}$ implies $\widehat{e} \rightarrow^{*} \widehat{e^{\prime}}$. As a consequence, if $e, e^{\prime} \in \operatorname{Exp}$ then $e \rightarrow^{l^{*}} e^{\prime}$ implies $e \rightarrow^{*} e^{\prime}$, i.e., $\left(\rightarrow^{l^{*}} \cap(\operatorname{Exp} \times \operatorname{Exp})\right) \subseteq \rightarrow^{*}$.

Proof

The first part follows from an immediate induction on the length of the let-derivation, using Lemma 13 for the inductive step. The rest is obvious taking into account that $e \equiv \widehat{e}$ and $e^{\prime} \equiv \widehat{e^{\prime}}$ when $e, e^{\prime} \in \operatorname{Exp}$.

To conclude this part, we can combine this last result with the equivalence of CRWL and let-rewriting, thus getting the following soundness result for CRWL with respect to term rewriting. 
Theorem 18 (Soundness of CRWL wrt. term rewriting)

For any $e \in \operatorname{Exp}, t \in C T e r m_{\perp}$, if $e \rightarrow t$ then there exists $e^{\prime} \in \operatorname{Exp}$ such that $e \rightarrow^{*} e^{\prime}$ and $t \sqsubseteq\left|e^{\prime}\right|$.

Proof

Assume $e \rightarrow t$. By Theorem 11 there exists $e^{\prime \prime} \in L \operatorname{Exp}$ such that $e \rightarrow^{l^{*}} e^{\prime \prime}$ and $t \sqsubseteq\left|e^{\prime \prime}\right|$. Then, by Theorem 17, we have $\widehat{e} \rightarrow^{*} \widehat{e^{\prime \prime}}$. As $e \in \operatorname{Exp}$, we have $e \equiv \widehat{e}$ and we can choose $e^{\prime} \equiv \widehat{e^{\prime \prime}} \in \operatorname{Exp}$ so we get $e \rightarrow^{*} e^{\prime}$. It is easy to check that $\left|e^{\prime \prime}\right|=\left|\widehat{e^{\prime \prime}}\right|$ and then we have $t \sqsubseteq\left|e^{\prime \prime}\right|=\left|\widehat{e^{\prime \prime}}\right|=\left|e^{\prime}\right|$.

\subsection{Completeness of CRWL wrt. classical term rewriting}

We prove here the completeness of the CRWL framework wrt. term rewriting for the class of CRWL-deterministic programs, which are defined as follows.

Definition 10 (CRWL-deterministic program)

A program $\mathcal{P}$ is $C R W L$-deterministic iff for any expression $e \in E x p_{\perp}$ its denotation $\llbracket e \rrbracket^{\mathcal{P}}$ is a directed set. In other words, iff for all $e \in \operatorname{Exp}_{\perp}$ and $t_{1}, t_{2} \in \llbracket e \rrbracket^{\mathcal{P}}$, there exists $t_{3} \in \llbracket e \rrbracket^{\mathcal{P}}$ with $t_{1} \sqsubseteq t_{3}$ and $t_{2} \sqsubseteq t_{3}$.

Thanks to the equivalence of CRWL and let-rewriting, it is easy to characterize CRWL-determinism also in terms of let-rewriting derivations.

Lemma 14

A program $\mathcal{P}$ is CRWL-deterministic iff for any $e \in \operatorname{Exp}, e^{\prime}, e^{\prime \prime} \in L E x p$ with $\mathcal{P} \vdash e \rightarrow^{l^{*}} e^{\prime}$ and $\mathcal{P} \vdash e \rightarrow^{l^{*}} e^{\prime \prime}$ there exists $e^{\prime \prime \prime} \in L \operatorname{Exp}$ such that $\mathcal{P} \vdash e \rightarrow^{l^{*}} e^{\prime \prime \prime}$ and $\left|e^{\prime \prime \prime}\right| \sqsupseteq\left|e^{\prime}\right|,\left|e^{\prime \prime \prime}\right| \sqsupseteq\left|e^{\prime \prime}\right|$.

\section{Proof}

For the left to right implication, assume a CRWL-deterministic program $\mathcal{P}$ and $e \in \operatorname{Exp}, e^{\prime}, e^{\prime \prime} \in L \operatorname{Exp}$ with $e \rightarrow^{l^{*}} e^{\prime}$ and $e \rightarrow^{l^{*}} e^{\prime \prime}$. By part iii) of Theorem 10 we have $\left|e^{\prime}\right|,\left|e^{\prime \prime}\right| \in \llbracket e \rrbracket$ and then by Definition 10 there exists $t \in \llbracket e \rrbracket$ such that $\left|e^{\prime}\right|,\left|e^{\prime \prime}\right| \sqsubseteq t$. Now, by part iii) of Theorem 11 there exists $e^{\prime \prime \prime} \in L E x p$ such that $e \rightarrow^{l^{*}} e^{\prime \prime \prime}$ and $t \sqsubseteq\left|e^{\prime \prime \prime}\right|$, so we have $\left|e^{\prime}\right|,\left|e^{\prime \prime}\right| \sqsubseteq t \sqsubseteq\left|e^{\prime \prime \prime}\right|$ as expected.

Regarding the converse implication, assume $e \in \operatorname{Exp}$ with $t_{1}, t_{2} \in \llbracket e \rrbracket$. By part iii) of Theorem 11 there exist $e^{\prime}, e^{\prime \prime} \in L$ Exp such that $e \rightarrow^{l^{*}} e^{\prime}, e \rightarrow l^{l^{*}} e^{\prime \prime}$ and $t_{1} \sqsubseteq\left|e^{\prime}\right|, t_{2} \sqsubseteq\left|e^{\prime \prime}\right|$. Then by hypothesis there exists $e^{\prime \prime \prime} \in L \operatorname{Exp}$ such that $e \rightarrow^{l^{*}} e^{\prime \prime \prime}$ and $\left|e^{\prime}\right|,\left|e^{\prime \prime}\right| \sqsubseteq\left|e^{\prime \prime \prime}\right|$. Now, by part iii) of Theorem 10 we have $\left|e^{\prime \prime \prime}\right| \in \llbracket e \rrbracket$ and this $\left|e^{\prime \prime \prime}\right|$ is the $t_{3}$ of Definition 10 we are looking for, i.e., $t_{3} \in \llbracket e \rrbracket$ and $t_{1}, t_{2} \sqsubseteq t_{3}$.

CRWL-determinism is intuitively close to confluence of term rewriting, but these two properties are not equivalent, as shown by the following example of a CRWLdeterministic but not confluent program. 
Example 7

Consider the program $\mathcal{P}$ given by the rules

$$
f \rightarrow a \quad f \rightarrow \text { loop } \quad \text { loop } \rightarrow \text { loop }
$$

where $a$ is a constructor. It is clear that $\rightarrow_{\mathcal{P}}$ is not confluent ( $f$ can be reduced to $a$ and loop, which cannot be joined into a common reduct), but it is CRWLdeterministic, since $\llbracket f \rrbracket^{\mathcal{P}}=\{\perp, a\}$, $\llbracket$ loop $\rrbracket^{\mathcal{P}}=\{\perp\}$ and $\llbracket a \rrbracket^{\mathcal{P}}=\{\perp, a\}$, which are all directed sets.

We conjecture that the reverse implication is true, i.e., that confluence of term rewriting implies CRWL-determinism. Nevertheless, a precise proof for this fact seems surprisingly complicated and we have not yet completed it.

A key ingredient in our completeness proof is the notion of CRWL-denotation of a substitution, which is the set of c-substitutions whose range can be obtained by CRWL-reduction over the range of the starting expression.

Definition 11 (CRWL-denotation for a substitution)

Given a program $\mathcal{P}$, the CRWL-denotation of a $\sigma \in S u b s t_{\perp}$ is defined as:

$$
\llbracket \sigma \rrbracket_{C R W L}^{\mathcal{P}}=\left\{\theta \in C S u b s t_{\perp} \mid \forall X \in \mathcal{V}, \mathcal{P} \vdash_{C R W L} \sigma(X) \rightarrow \theta(X)\right\}
$$

We will usually omit the subscript CRWL and/or the superscript $\mathcal{P}$ when implied by the context.

Any substitution $\theta$ in the denotation of some substitution $\sigma$ contains less information than $\sigma$, because it only holds in its range a finite part of the possibly infinite denotation of the expressions in the range of $\sigma$. We formalize this property in the following result.

Proposition 10

For all $\sigma \in S u b s t_{\perp}, \theta \in \llbracket \sigma \rrbracket$, we have that $\theta \unlhd \sigma$.

Besides, we will use the notion of deterministic substitution, which is a substitution with only deterministic expressions in its range.

Definition 12 (Deterministic substitution)

The set $D S u b s t_{\perp}$ of deterministic substitutions for a given program $\mathcal{P}$ is defined as

$$
\text { DSubst }_{\perp}=\left\{\sigma \in \text { Subst }_{\perp} \mid \forall X \in \operatorname{dom}(\sigma) \cdot \llbracket \sigma(X) \rrbracket \text { is a directed set }\right\}
$$

Then $C S u b s t_{\perp} \subseteq D S u b s t_{\perp}$, and under any program $\forall \sigma \in S u b s t_{\perp} \cdot \llbracket \sigma \rrbracket \subseteq C S u b s t_{\perp} \subseteq$ DSubst $\perp_{\perp}$. Note that the determinism of substitutions depends on the program, which gives meaning to the functions in its range. Obviously if a program is deterministic then $S u b s t_{\perp}=D S u b s t_{\perp}$.

A good thing about deterministic substitutions is that their denotation is always a directed set. 
Proposition 11

For all $\sigma \in D S u s b t_{\perp}, \llbracket \sigma \rrbracket$ is a directed set.

But the fundamental property of deterministic substitutions is that, for any CRWL-statement starting from an instance of an expression that has been constructed using a deterministic substitution, there is another CRWL-statement to the same value from another instance of the same expression that now has been built using a c-substitution taken from the denotation of the starting substitution. This property is a direct consequence of Proposition 11.

Lemma 15

For all $\sigma \in D S u s b t_{\perp}, e \in \operatorname{Exp}_{\perp}, t \in C T e r m_{\perp}$,

$$
\text { if } e \sigma \rightarrow t \text { then } \exists \theta \in \llbracket \sigma \rrbracket \text { such that } e \theta \rightarrow t
$$

Proof (sketch)

We proceed by a case distinction over $e$. If $e \equiv X \in \operatorname{dom}(\sigma)$ then we have $e \sigma \equiv$ $\sigma(X) \rightarrow t$, and we can define

$$
\theta(Y)= \begin{cases}t & \text { if } Y \equiv X \\ \perp & \text { if } Y \in \operatorname{dom}(\sigma) \backslash\{X\} \\ Y & \text { otherwise }\end{cases}
$$

Then it is easy to check that $\theta \in \llbracket \sigma \rrbracket$ and besides $e \theta \equiv \theta(X) \equiv t \rightarrow t$ by Lemma 5 so we are done. If $e \equiv X \in \mathcal{V} \backslash \operatorname{dom}(\sigma)$ then we have $e \sigma \equiv \sigma(X) \equiv X \rightarrow t$, and given $\bar{Y}=\operatorname{dom}(\sigma)$ it is easy to check that $[\overline{Y / \perp}] \in \llbracket \sigma \rrbracket$, and besides $e[\overline{Y / \perp}] \equiv X \rightarrow t$ by hypothesis.

Finally if $e \notin \mathcal{V}$ we proceed by induction on the structure of the proof for $e \sigma \rightarrow t$. The interesting cases are those for (DC) and (OR) where we use that $\sigma \in D S u s b t_{\perp}$, so by Proposition 11 its denotation is directed. Then there must exist some $\theta \in$ $\llbracket \sigma \rrbracket$ which is greater than each of the $\theta_{i}$ obtained by induction hypothesis over the premises of the starting CRWL-proof for $e \sigma \rightarrow t$. Using the monotonicity of Proposition 5 we can prove $e \theta \rightarrow t$, which also holds for CRWL, by Theorem 4 see the online appendix, page 38 for details.

Now we are finally ready to prove our first completeness result of CRWL wrt. term rewriting, for deterministic programs.

Lemma 16 (Completeness lemma for CRWL wrt. term rewriting) Let $\mathcal{P}$ be a CRWL-deterministic program, and $e, e^{\prime} \in \operatorname{Exp}$. Then:

$$
e \rightarrow^{*} e^{\prime} \text { implies } \llbracket e^{\prime} \rrbracket \subseteq \llbracket e \rrbracket
$$

Proof

We can just prove this result for $e \rightarrow e^{\prime}$, then its extension for an arbitrary number of term rewriting steps holds by a simple induction on the length of the term rewriting derivation, using transitivity of $\subseteq$.

Assume $e \rightarrow e^{\prime}$, then the step must be of the shape $e \equiv \mathcal{C}[f(\bar{p}) \sigma] \rightarrow \mathcal{C}[r \sigma] \equiv e^{\prime}$ for some program rule $(f(\bar{p}) \rightarrow r) \in \mathcal{P}, \sigma \in$ Subst. First, let us focus on the case for 
$\mathcal{C}=[]$, and then assume some $t \in C$ Term ${ }_{\perp}$ such that $\mathcal{P} \vdash_{C R W L} e^{\prime} \equiv \operatorname{ro} \rightarrow t$. As $\mathcal{P}$ is deterministic then $\sigma \in D S u b s t_{\perp}$, therefore by Lemma 15 there must exist some $\theta \in \llbracket \sigma \rrbracket$ such that $\mathcal{P} \vdash_{C R W L} r \theta \rightarrow t$. But then we can use $\theta$ to build the following CRWL-proof.

$$
\frac{\ldots p_{i} \theta \rightarrow p_{i} \theta \ldots r \theta \rightarrow t}{f(\bar{p}) \theta \rightarrow t} O R
$$

where for each $p_{i} \in \bar{p}$ we have $\mathcal{P} \vdash_{C R W L} p_{i} \theta \rightarrow p_{i} \theta$ by Lemma 5 as $p_{i} \in C$ Term because $\mathcal{P}$ is a constructor system, and so $p_{i} \theta \in C T e r m_{\perp}$, as $\theta \in \llbracket \sigma \rrbracket \subseteq C S u b s t_{\perp}$. But we also have $\theta \unlhd \sigma$ by Proposition 10 , therefore by applying the monotonicity for substitutions from Proposition 5 - which also holds for CRWL, by Theorem 4 we get $\mathcal{P} \vdash_{C R W L} e \equiv f(\bar{p}) \sigma \rightarrow t$. Hence $\llbracket e^{\prime} \rrbracket=\llbracket r \sigma \rrbracket \subseteq \llbracket f(\bar{p}) \sigma \rrbracket=\llbracket e \rrbracket$.

Finally, we can generalize this result to arbitrary contexts by using the compositionality of CRWL from Theorem 1. Given a term rewriting step $e \equiv \mathcal{C}[f(\bar{p}) \sigma] \rightarrow$ $\mathcal{C}[r \sigma] \equiv e^{\prime}$ then by the proof for $\mathcal{C}=[]$ we get $\llbracket r \sigma \rrbracket \subseteq \llbracket f(\bar{p}) \sigma \rrbracket$, but then

$$
\begin{array}{ll}
\llbracket e^{\prime} \rrbracket=\llbracket \mathcal{C}[r \sigma] \rrbracket & \\
=\bigcup_{t \in \llbracket r \sigma \rrbracket} \llbracket \mathcal{C}[t] \rrbracket & \text { by Theorem } 1 \\
\subseteq \bigcup_{t \in \llbracket f(\bar{p}) \sigma \rrbracket} \llbracket \mathcal{C}[t] \rrbracket & \text { as } \llbracket r \sigma \rrbracket \subseteq \llbracket f(\bar{p}) \sigma \rrbracket \\
=\llbracket \mathcal{C}[f(\bar{p}) \sigma] \rrbracket=\llbracket e \rrbracket & \text { by Theorem } 1
\end{array}
$$

The previous lemma, together with the equivalence of CRWL and let-rewriting given by Theorem 12 and Theorem 4 , allows us to obtain a strong relationships between term rewriting, let-rewriting and CRWL, for the class of CRWL-deterministic programs.

Theorem 19

Let $\mathcal{P}$ be a CRWL-deterministic program, and $e, e^{\prime} \in \operatorname{Exp}, t \in C T e r m$. Then:

a) $e \rightarrow^{*} e^{\prime}$ implies $e \rightarrow^{l^{*}} e^{\prime \prime}$ for some $e^{\prime \prime} \in L \operatorname{Exp}$ with $\left|e^{\prime \prime}\right| \sqsupseteq\left|e^{\prime}\right|$.

b) $e \rightarrow^{*} t$ iff $e \rightarrow^{l^{*}} t$ iff $\mathcal{P} \vdash_{C R W L} e \rightarrow t$.

Notice that in part $a$ ) we cannot ensure $e \rightarrow^{*} e^{\prime}$ implies $e \rightarrow^{l^{*}} e^{\prime}$, because term rewriting can reach some intermediate expressions not reachable by let-rewriting. For instance, given the deterministic program with the rules $g \rightarrow a$ and $f(x) \rightarrow$ $c(x, x)$, we have $f(g) \rightarrow{ }^{*} c(g, a)$, but $f(g) \not \rightarrow^{l^{*}} c(g, a)$. Still, parts $\left.a\right)$ is a strong completeness results for let-rewriting wrt. term rewriting for deterministic programs, since it says that the outer constructed part obtained in a rewriting derivation can be also obtained or even refined in a let-rewriting derivation. Combined with Theorem 17, part a) expresses a kind of equivalence between let-rewriting and term rewriting, valid for general derivations, even non-terminating ones. For derivations reaching a constructor term (not further reducible), part b) gives an even stronger equivalence result. 


\subsection{Let-narrowing versus narrowing for deterministic systems}

Joining the results of the previous section with the adequacy of let-narrowing to let-rewriting, we can easily establish some relationships between let-narrowing and ordinary term rewriting/narrowing, summarized in the following result.

Theorem 20

For any program $\mathcal{P}, e \in E x p, \theta \in C S u b s t$ and $t \in C$ Term:

a) If $e \rightsquigarrow l_{\theta}^{*} t$ then $e \theta \rightarrow^{*} t$.

b) If in addition $\mathcal{P}$ is CRWL-deterministic, then:

$\mathbf{b}_{1}$ ) If $e \theta \rightarrow^{*} t$ then $\exists t^{\prime} \in C$ Term, $\sigma, \theta^{\prime} \in C S u b s t$ such that $e \rightsquigarrow \underset{\sigma}{l^{*}} t^{\prime}, t^{\prime} \theta^{\prime} \equiv t$ and $\sigma \theta^{\prime}=\theta[\operatorname{var}(e)]$.

$\mathbf{b}_{2}$ ) If $e \rightsquigarrow{ }_{\theta}^{*} t$, the same conclusion of $\left(b_{1}\right)$ holds.

Part a) expresses soundness of $\rightsquigarrow^{l}$ wrt. term rewriting, and part $b$ ) is a completeness result for $\rightsquigarrow^{l}$ wrt. term rewriting/narrowing, for the class of deterministic programs.

Proof

Part a) follows from soundness of let-narrowing wrt. let-rewriting (Theorem 14 and soundness of let-rewriting wrt. term rewriting of Theorem 19 .

For part $\left.b_{1}\right)$, for let-narrowing, assume $e \theta \rightarrow^{*} t$. By the completeness of letrewriting wrt. term rewriting for deterministic programs (Theorem 19), we have $e \theta \rightarrow{ }^{l}{ }^{*} t$, and then by the completeness of let-narrowing wrt. let-rewriting (Theorem 15 , there exists a narrowing derivation $e \rightsquigarrow{ }_{\sigma}^{*} t^{\prime}$ with $t^{\prime} \theta^{\prime}=t$ and $\sigma \theta^{\prime}=\theta[F V(e)]$. But notice that for $e \in \operatorname{Exp}$, the sets $F V(e)$ and $\operatorname{var}(e)$ coincide, and the proof is finished.

Finally, $b_{2}$ ) follows simply from soundness of (ordinary) narrowing wrt. term rewriting and $\left.b_{1}\right)$.

\section{Conclusions}

This paper contains a thorough presentation of the theory of first order let-rewriting and let-narrowing for constructor-based term rewriting systems. These two relations are simple notions of one-step reduction that express sharing as it is required by the call-time choice semantics of non-determinism adopted in the functional logic programming paradigm. In a broad sense, let-rewriting and let-narrowing can be seen as particular syntactical presentations of term graph rewriting and narrowing. However, keeping our formalisms very close to the syntax and basic notions of term rewriting systems (terms, substitutions, syntactic unification,... ) has been an essential aid in establishing strong equivalence results with respect to the CRWLframework - a well-established realization of call-time choice semantics-, which was one of the main aims of the paper.

Along the way of proving such equivalence we have developed powerful semantic tools that are interesting in themselves. Most remarkably, the $\mathrm{CRWL}_{l e t}$-logic, a 
conservative extension of CRWL that deals with let-bindings, and the notion of hypersemantics of expressions and contexts, for which we prove deep compositionality results not easily achievable by thinking directly in terms of reduction sequences.

We have shown in several places the methodological power of having provably equivalent reduction-based and logic-based semantics. In some occasions, we have used the properties of the CRWL-semantics to investigate interesting aspects of reductions, as replaceability conditions or derived operational rules, like bubbling. In others, we have followed the converse way. For instance, by transforming letrewriting reductions into ordinary term rewriting reductions, we easily concluded that let-rewriting (call-time choice) provides less computed values than term rewriting (run-time choice). By using again semantic methods, we proved the opposite inclusion for deterministic programs, obtaining for such programs an equivalence result of let-rewriting and term rewriting.

In our opinion, the different pieces of this work can be used separately for different purposes. The $\mathrm{CRWL}_{l e t}$-logic provides a denotational semantics reflecting call-time choice for programs making use of local bindings. The let-rewriting and let-narrowing relations provide clear and abstract descriptions of how computations respecting call-time choice can proceed. They can be useful to explain basic operational aspects of functional logic languages to students or novice programmers, for instance. They have been used also as underlying formalisms to investigate other aspects of functional logic programming that need a clear notion of reduction; for instance, when proving essential properties of type systems, like subject reduction or progress. In addition, all the pieces are interconnected by strong theoretical results, which may be useful depending on the pursued goal.

Just like classical term rewriting and narrowing, the let-rewriting and narrowing relations define too broad computation spaces as to be adopted directly as concrete operational procedures of a programming language. To that purpose, they should be accompanied by a strategy that selects only certain computations. In this paper we have only given an example-driven discussion of strategies. We are quite confident that some known on-demand evaluation strategies, like lazy, needed or natural rewriting/narrowing, can be adapted to our formal setting. In (Riesco and Rodríguez-Hortalá 2010: Sánchez-Hernández 2011) we work out in more detail two concrete on-demand strategies for slight variants of let-rewriting and narrowing formalisms.

A subject of future work that might be of interest to the functional logic community is that of completing the comparison of different formalisms proposed in the field to capture call-time choice semantics: CRWL, admissible term graph rewriting/narrowing, natural semantics à la Launchbury, and let-rewriting/narrowing. Proving their equivalence would greatly enrich the set of tools available to the functional logic programming theoretician, since any known or future result obtained for one of the approaches could be applied to the rest on a sound technical basis. 


\section{References}

Albert, E., Hanus, M., Huch, F., Oliver, J., and Vidal, G. 2005. Operational semantics for declarative multi-paradigm languages. Journal of Symbolic Computation 40, 1, $795-829$

Alpuente, M., Falaschi, M., Iranzo, P. J., and Vidal, G. 2003. Uniform lazy narrowing. Journal of Logig and Computation 13, 2, 287-312.

ANTOY, S. 2005. Evaluation strategies for functional logic programming. Journal of Symbolic Computation 40, 1, 875-903.

Antoy, S., Brown, D., And Chiang, S. 2006. On the correctness of bubbling. In 17th International Conference on Rewriting Techniques and Applications (RTA'06). Springer LNCS 4098, 35-49.

Antoy, S., Brown, D., And ChiAng, S. 2007. Lazy context cloning for non-deterministic graph rewriting. Electronic Notes in Theoretical Computer Science 176, 1, 3-23.

Antoy, S., Echahed, R., And Hanus, M. 1994. A needed narrowing strategy. In 21st ACM SIGPLAN-SIGACT Symposium on Principles of Programming Languages (POPL'94). ACM, 268-279.

Antoy, S., Echahed, R., AND Hanus, M. 2000. A needed narrowing strategy. Journal of the ACM 47, 4, 776-822.

Antoy, S. AND Hanus, M. 2000. Compiling multi-paradigm declarative programs into prolog. In 3rd International Workshop on Frontiers of Combining Systems (FroCoS'00). Springer LNCS 1794, 171-185.

Antoy, S. AND Hanus, M. 2006. Overlapping rules and logic variables in functional logic programs. In 22nd International Conference on Logic Programming (ICLP'06). Springer LNCS 4079, 87-101.

Ariola, Z. M. AND ARvind. 1995. Properties of a first-order functional language with sharing. Theoretical Computer Science 146, 1832, 69-108.

Ariola, Z. M. AND Felleisen, M. 1997. The call-by-need lambda calculus. Journal of Functional Programming 7, 3, 265-301.

Ariola, Z. M., Felleisen, M., Maraist, J., Odersky, M., and Wadler, P. 1995. The call-by-need lambda calculus. In 22nd ACM SIGPLAN-SIGACT Symposium on Principles of Programming Languages (POPL'95). ACM, 233-246.

BaAder, F. And Nipkow, T. 1998. Term Rewriting and All That. Cambridge University Press.

Barendregt, H. P., Eekelen, M. C. J. D., Glauert, J. R. W., Kennaway, J. R., Plasmeijer, M. J., And Sleep, M. R. 1987. Term Graph Rewriting. In 1st Parallel Architectures and Languages Europe (PARLE'87), Volume II. Springer LNCS 259, 141158 .

BRASSEL, B. AND HuCH, F. 2007. On a tighter integration of functional and logic programming. In 5th Asian Symposium on Programming Languages and Systems (APLAS'07). Springer LNCS 4807, 122-138.

Caballero, R. And SÁnchez, J., Eds. 2006. TOY: A multiparadigm declarative language, version 2.2.3. Technical report, Universidad Complutense de Madrid.

Cheong, P. And Fribourg, L. 1993. Implementation of narrowing: The Prolog-based approach. In Logic programming languages: constraints, functions, and objects. MIT Press, 1-20.

DeGroot, D. And Lindstrom, G. E. 1986. Logic Programming, Functions, Relations, and Equations. Prentice Hall.

Dios-Castro, J. and López-Fraguas, F. J. 2007. Extra variables can be eliminated 
from functional logic programs. Electronic Notes in Theoretical Computer Science 188 188, 3-19.

Echahed, R. And Janodet, J.-C. 1997. On constructor-based graph rewriting systems. Research Report 985-I, IMAG.

Echahed, R. And JANOdet, J.-C. 1998. Admissible graph rewriting and narrowing. In Joint International Conference and Symposium on Logic Programming (JICSLP'96). MIT Press, 325 - 340.

Escobar, S., Meseguer, J., And Thati, P. 2005. Natural narrowing for general term rewriting systems. In 16th International Conference on Rewriting Techniques and Applications (RTA'05). Springer LNCS 3467, 279-293.

González-Moreno, J. C., Hortalá-González, T., López-Fraguas, F. J., And Rodríguez-Artalejo, M. 1996. A rewriting logic for declarative programming. In 6th European Symposium on Programming (ESOP'96). Springer LNCS 1058, 156-172.

GonzÁlez-Moreno, J. C., Hortalá-González, T., López-Fraguas, F. J., And Rodríguez-Artalejo, M. 1999. An approach to declarative programming based on a rewriting logic. Journal of Logic Programming 40, 1, 47-87.

González-Moreno, J. C., Hortalá-González, T., and Rodríguez-Artalejo, M. 1997. A higher order rewriting logic for functional logic programming. In 14th International Conference on Logic Programming (ICLP'97). MIT Press, 153-167.

Hanus, M. 1994. The integration of functions into logic programming: From theory to practice. Journal of Logic Programming 198320, 583-628.

Hanus, M. 2007. Multi-paradigm declarative languages. In 23rd International Conference on Logic Programming (ICLP'07). Springer LNCS 4670, 45-75.

Hanus, M., Kuchen, H., And Moreno-Navarro, J. J. 1995. Curry: A truly functional logic language. In Workshop on Visions for the Future of Logic Programming (ILPS'95). 95-107.

Hanus, M., Ed. 2006. Curry: An integrated functional logic language (version 0.8.2). Available at http://www.informatik.uni-kiel.de/ curry/report.html.

Hullot, J. 1980. Canonical forms and unification. In 5th Conference on Automated Deduction (CADE'80). Springer LNCS 87, 318-334.

Hussmann, H. 1993. Non-Determinism in Algebraic Specifications and Algebraic Programs. Birkhäuser Verlag.

Kutzner, A. AND Schmidt-Schauss, M. 1998. A non-deterministic call-by-need lambda calculus. In 3th ACM SIGPLAN International Conference on Functional Programming (ICFP'98). ACM SIGPLAN Notices 34(1), 324-335.

LAunchBury, J. 1993. A natural semantics for lazy evaluation. In 20th ACM SIGPLANSIGACT Symposium on Principles of Programming Languages (POPL'93). ACM, 144154.

Loogen, R., López-Fraguas, F. J., And Rodríguez-Artalejo, M. 1993. A demand driven computation strategy for lazy narrowing. In 5th International Symposium on Programming Language Implementation and Logic Programming (PLILP'93). Springer LNCS 714, 184-200.

López-Fraguas, F. J., Martin-Martin, E., and Rodríguez-Hortalá, J. 2010a. Liberal typing for functional logic programs. In 8th Asian Symposium on Programming Languages and Systems (APLAS'10). Springer LNCS 6461, 80-96.

López-Fraguas, F. J., Martin-Martin, E., And Rodríguez-Hortalá, J. 2010b. New results on type systems for functional logic programming. In 18th International Workshop on Functional and (Constraint) Logic Programming (WFLP'09), Revised Selected Papers. Springer LNCS 5979, 128-144. 
López-Fraguas, F. J. And Rodríguez-Hortalá, J. 2010. The full abstraction problem for higher order functional-logic programs. CoRR, arXiv:1002:1833.

López-Fraguas, F. J., Rodríguez-Hortalá, J., And Sánchez-Hernández, J. 2007a. Equivalence of two formal semantics for functional logic programs. Electronic Notes in Theoretical Computer Science 188 188, 117-142.

López-Fraguas, F. J., Rodríguez-Hortalá, J., And SÁnchez-Hernández, J. 2007b. A simple rewrite notion for call-time choice semantics. In 9th International Conference on Principles and Practice of Declarative Programming (PPDP'07). ACM, 197-208.

López-Fraguas, F. J., Rodríguez-Hortalá, J., and Sánchez-Hernández, J. 2008. Rewriting and call-time choice: the HO case. In 9th International Symposium on Functional and Logic Programming (FLOPS'08). Springer LNCS 4989, 147-162.

López-Fraguas, F. J., Rodríguez-Hortalá, J., And SÁnchez-Hernández, J. 2009a. A flexible framework for programming with non-deterministic functions. In 2009 ACM SIGPLAN Symposium on Partial Evaluation and Program Manipulation (PEPM'09). ACM, 91-100.

López-Fraguas, F. J., Rodríguez-Hortalá, J., And SÁnchez-Hernández, J. 2009b. A fully abstract semantics for constructor based term rewriting systems. In 20th International Conference on Rewriting Techniques and Applications (RTA'09). Springer LNCS 5595, 320-334.

López-Fraguas, F. J., Rodríguez-Hortalá, J., And SÁnchez-Hernández, J. 2009c. Narrowing for First Order Functional Logic Programs with Call-Time Choice Semantics. In 17th International Conference on Applications of Declarative Programming and Knowledge Management (INAP'O'7) and 21st Workshop on (Constraint) Logic Programming (WLP'07), Revised Selected Papers. Springer LNAI 5437, 206-222.

López-Fraguas, F. J. And SÁnchez-Hernández, J. 1999. TOYY: A multiparadigm declarative system. In 10th International Conference on Rewriting Techniques and Applications (RTA'99). Springer LNCS 1631, 244-247.

López-Fraguas, F. J. And SÁnchez-Hernández, J. 2001. Functional logic programming with failure: A set-oriented view. In 8th International Conference on Logic for Programming and Automated Reasoning (LPAR'01). Springer LNAI 2250, 455-469.

Maraist, J., Odersky, M., And Wadler, P. 1998. The call-by-need lambda calculus. Journal of Functional Programming 8, 3, 275-317.

McCarthy, J. 1963. A Basis for a Mathematical Theory of Computation. In Computer Programming and Formal Systems. North-Holland, 33-70.

Moreno-Navarro, J. J. And Rodríguez-Artalejo, M. 1992. Logic programming with functions and predicates: The language Babel. Journal of Logic Programming 12, 189223.

Plump, D. 1998. Term graph rewriting. Report CSI-R9822, Computing Science Institute, University of Nijmegen.

Plump, D. 2001. Essentials of term graph rewriting. Electronic Notes Theoretical Computer Science 51, 277-289.

Riesco, A. ANd Rodríguez-Hortalá, J. 2010. Programming with singular and plural non-deterministic functions. In 2010 ACM SIGPLAN Workshop on Partial Evaluation and Program Manipulation (PEPM'10). ACM, 83-92.

Rodríguez-Artalejo, M. 2001. Functional and constraint logic programming. In Revised Lectures of the International Summer School CCL'99. Springer LNCS 2002, 202-270.

SÁNCHEZ-HERNÁNDEZ, J. 2004. Una aproximación al fallo constructivo en programación declarativa multiparadigma. Ph.D. thesis, Departamento Sistemas Informáticos y Programación, Universidad Complutense de Madrid. 
SÁNCHEZ-HERnÁNDEZ, J. 2011. Reduction strategies for rewriting with call-time choice. In 11th Jornadas sobre Programación y Lenguajes (PROLE'11).

SchmidT-Schauss, M. And MachKasova, E. 2008. A finite simulation method in a nondeterministic call-by-need lambda-calculus with letrec, constructors, and case. In 19th International Conference on Rewriting Techniques and Applications (RTA'08). Springer LNCS 5117, 321-335.

Sondergand, H. And Sestoft, P. 1990. Referential transparency, definiteness and unfoldability. Acta Informatica 27, 6, 505-517.

SøndergaArd, H. And Sestoft, P. 1992. Non-determinism in functional languages. The Computer Journal 35, 5, 514-523.

VAdo-VÍRsedA, R. D. 2002. Estrategias de estrechamiento perezoso. Trabajo de Investigación de Tercer Ciclo, Dpto. de Sistemas Informáticos y Programación, Universidad Complutense de Madrid.

VADO-VÍRSEDA, R. D. 2003. A demand-driven narrowing calculus with overlapping definitional trees. In 5th ACM SIGPLAN Conference on Principles and Practice of Declarative Programming (PPDP'03). ACM, 213-227.

Zartmann, F. 1997. Denotational abstract interpretation of functional logic programs. In 4 th International Symposium on Static Analysis (SAS'97). Springer LNCS 1302, $141-159$. 